Draft Version OCtOBER 30, 2018

Preprint typeset using $\mathrm{L}_{\mathrm{A}} \mathrm{T} \mathrm{X}$ style emulateapj v. 2/16/10

\title{
SUBMILLIMETRE LINE SPECTRUM OF THE SEYFERT GALAXY NGC 1068 FROM THE Herschel-SPIRE FOURIER TRANSFORM SPECTROMETER *
}

Luigi Spinoglio ${ }^{1}$, Miguel Pereira-Santaella ${ }^{1}$, Gemma Busquet ${ }^{1}$, Maximilien R. P. Schirm ${ }^{2}$, Christine D. Wilson $^{2}$, Jason Glenn ${ }^{3}$, Julia Kamenetzky ${ }^{3}$, Naseem Rangwala ${ }^{3}$, Philip R. Maloney ${ }^{3}$, Tara J. Parkin ${ }^{2}$, George J. Bendo ${ }^{4}$, Suzanne C. Madden ${ }^{5}$, Mark G. Wolfire $^{6}$, Alessandro Bosellit ${ }^{7}$, Asantha Cooray ${ }^{8}$, Mathew J. Page ${ }^{9}$

Draft version October 30, 2018

The first complete submillimetre spectrum $(190-670 \mu \mathrm{m})$ of the Seyfert 2 galaxy NGC 1068 has been observed with the SPIRE Fourier Transform Spectrometer onboard the Herschel Space Observatory. The sequence of $\mathrm{CO}$ lines $\left(\mathrm{J}_{u p}=4-13\right)$, lines from $\mathrm{H}_{2} \mathrm{O}$, the fundamental rotational transition of $\mathrm{HF}$, two o- $\mathrm{H}_{2} \mathrm{O}^{+}$lines and one line each from $\mathrm{CH}^{+}$and $\mathrm{OH}^{+}$have been detected, together with the two $[\mathrm{CI}]$ lines and the [NII] $205 \mu \mathrm{m}$ line. The observations in both single pointing mode with sparse image sampling and in mapping mode with full image sampling allow us to disentangle two molecular emission components, one due to the compact circum-nuclear disk (CND) and one from the extended region encompassing the star forming ring (SF-ring). Radiative transfer models show that the two CO components are characterized by density of $n\left(\mathrm{H}_{2}\right)=10^{4.5}$ and $10^{2.9} \mathrm{~cm}^{-3}$ and temperature of $T_{\text {kin }}$ $=100 \mathrm{~K}$ and $127 \mathrm{~K}$, respectively. The comparison of the $\mathrm{CO}$ line intensities with photodissociation region (PDR) and X-ray dominated region (XDR) models, together with other observational constraints, such as the observed $\mathrm{CO}$ surface brightness and the radiation field, indicate that the best explanation for the $\mathrm{CO}$ excitation of the CND is an XDR with density of $\mathrm{n}\left(\mathrm{H}_{2}\right) \sim 10^{4} \mathrm{~cm}^{-3}$ and X-ray flux of $9 \mathrm{erg} \mathrm{s}^{-1} \mathrm{~cm}^{-2}$, consistent with illumination by the active galactic nucleus, while the $\mathrm{CO}$ lines in the SF-ring are better modeled by a PDR. The detected water transitions, together with those observed with the Herschel PACS Spectrometer, can be modeled by an LVG model with low temperature $\left(T_{\text {kin }} \sim 40 \mathrm{~K}\right)$ and high density $\left(n\left(\mathrm{H}_{2}\right)\right.$ in the range $\left.10^{6.7}-10^{7.9} \mathrm{~cm}^{-3}\right)$. The emission of $\mathrm{H}_{2} \mathrm{O}^{+}$and $\mathrm{OH}^{+}$are in agreement with PDR models with cosmic ray ionization. The diffuse ionized atomic component observed through the [NII] $205 \mu \mathrm{m}$ line is consistent with previous photoionization models of the starburst.

Subject headings: Galaxies: individual: NGC 1068 - Galaxies: ISM, nuclei, active, starburst, Seyfert - Techniques: imaging spectroscopy

\section{INTRODUCTION}

NGC 1068 (Messier 77) is a nearby $(c z=1137 \mathrm{~km}$ $\left.\mathrm{s}^{-1}\right)$ and bright $\left(\mathrm{L}_{I R}=\mathrm{L}_{8-1000 \mu m} \sim 2 \times 10^{11} \mathrm{~L}_{\odot}\right.$, BlandHawthorn et al. 1997) Seyfert galaxy, often considered as the prototypical Seyfert type 2 galaxy. However, since the discovery of the broad permitted lines in the polarized optical spectrum of NGC 1068 (Antonucci \& Miller

\section{luigi.spinoglio@iaps.inaf.it}

* Herschel is an ESA space observatory with science instruments provided by European-led Principal Investigator consortia and with important participation from NASA.

${ }^{1}$ Istituto di Astrofisica e Planetologia Spaziali, INAF-IAPS,

Via Fosso del Cavaliere 100, I-00133 Roma, Italy

2 Dept. of Physics \& Astronomy, McMaster University, Hamilton, Ontario, L8S 4M1, Canada

${ }^{3}$ Center for Astrophysics and Space Astronomy, 389-UCB, University of Colorado, Boulder, CO, 80303

${ }^{4}$ UK ALMA Regional Centre Node, Jordell Bank Center for Astrophysics, School of Physics and Astronomy, University of Manchester, Oxford Road, Manchester M13 9PL, U.K.

${ }^{5}$ CEA, Laboratoire AIM, Irfu/SAp, Orme des Merisiers, 91191 Gif-sur-Yvette, France

${ }^{6}$ Astronomy Department, University of Maryland, College Park, MD 20742, USA

${ }^{7}$ Laboratoire d'Astrophysique de Marseille - LAM, Université d'Aix-Marseille \& CNRS, UMR7326, 38 rue F. Joliot-Curie, 13388, Marseille Cedex 13

${ }^{8}$ Center for Cosmology, Department of Physics and Astronomy, University of California, Irvine, CA 92697

${ }^{9}$ Mullard Space Science Laboratory, University College London, Holmbury St Mary, Dorking, Surrey RH5 6NT
1985), it has become clear that this galaxy was in reality a hidden broad line region galaxy. The general distinction between the two types of Seyfert galaxies might be due only to orientation effects, according to the so called Unification model (Antonucci 1993).

Being the strongest nearby Seyfert 2 galaxy, it has been observed extensively over the whole electromagnetic spectrum. Molecular ( $\mathrm{CO}$ and $\mathrm{HCN}$ ) observations have shown a prominent starburst ring (hereafter SF-ring) at a radius of 1.0-1.5 kpc and a central circum-nuclear disk (hereafter CND) with a diameter of $d \sim 300$ pc (Tacconi et al. 1994; Schinnerer et al. 2000). Near-IR observations (Scoville et al. 1988, Thronson et al. 1989) have clearly revealed a $2.3 \mathrm{kpc}$ stellar bar. The compact $(\sim 1 \mathrm{pc})$ hot dust source in the nucleus of NGC 1068, measured using near-infrared speckle imaging and integral field spectroscopy (Thatte et al. 1997), is probably heated by the AGN's strong radiation field and possibly associated with the postulated dense circum-nuclear torus. A jet was observed from centimeter to millimeter wavelengths extending out to several kiloparsecs from the center (e.g., Krips et al. 2006, Gallimore et al. 2004). Mid-IR observations revealed hot and ionized gas biconically following the path of the radio jet (e.g., Müller Sánchez et al. 2009. and references therein) and indicating the existence of a parsec-scale warm dust torus (Jaffe et al. 2004). Recent interferometric observations of $\mathrm{CO}(3-2)$ and $\mathrm{CO}(1-0)$ 
show that the $\mathrm{CO}(3-2)$ emission peaks in the central region within $\sim 5^{\prime \prime}$ from the nucleus, while the $\mathrm{CO}(1-0)$ emission is mainly located along the spiral arms (Tsai et al. 2012).

Spectroscopic coverage of NGC 1068 has been extensive. In the mid-IR to far-IR wavelength range, spectra have been measured by the ISO (Kessler et al.1996) SWS (de Graauw et al. 1996) and LWS (Clegg et al. 1996) spectrometers (Lutz et al. 2000, Spinoglio et al. 2005 respectively), covering the $2.4-45 \mu \mathrm{m}$ and $43-197 \mu \mathrm{m}$ spectral ranges. At millimeter wavelengths it was recently observed from the ground in the $190-307 \mathrm{GHz}$ (976-1578 $\mu \mathrm{m})$ range (Kamenetzky et al. 2011). The submillimeter waveband is one of the few spectral regions that have not been so far explored; the new observations made by the Spectral and Photometric Imaging Receiver (SPIRE) (Griffin et al.2010) Fourier Transform Spectrometer (FTS) (Naylor et al. 2010b), onboard the Herschel Space Observatory (Pilbratt et al. 2010), covering the spectral range from $190 \mu \mathrm{m}$ to $670 \mu \mathrm{m}$, fill most of this gap with the first complete submillimeter spectrum of NGC 1068.

Submillimeter spectral measurements are of particular interest as NGC 1068 is a prime candidate to study the effects of the AGN onto the circum-nuclear material and the surrounding disk. In particular, the study of the excitation conditions and chemistry of the CND appear already from the existing ground-based molecular line observations to be very different from the starburst galaxies environments. The peculiar line ratios of different molecular transitions, mostly $\mathrm{HCN}, \mathrm{HCO}^{+}$, and ${ }^{12} \mathrm{CO}$, led to the suggestion that the CND of NGC 1068 harbors a giant X-ray-dominated region (XDR, e.g., Rotaciuc et al. 1991, Maloney et al. 1996; Usero et al. 2004; Kohno et al. 2008). The $\mathrm{HCN}$ and $\mathrm{HCO}^{+}$molecular line studies of Krips et al. (2008, 2011) confirm an increased abundance of $\mathrm{HCN}$ and/or increased kinetic temperatures. CO lines can also discriminate between "classical" photodissociation regions (PDRs) and X-ray dominated regions (XDRs) (e.g. Meijerink \& Spaans 2005). Using the intermediate $\mathrm{J}$ rotational lines from the $\mathrm{CO}$ molecule, from $\mathrm{J}_{u p}=4$ to $\mathrm{J}_{u p}=13$, which can be observed with the SPIRE FTS, we want to test if indeed in NGC 1068 an $\mathrm{XDR}$ is needed to explain the spectral line energy distribution originating from the CND. The case of the ultraluminous IR galaxy Mrk231 already demonstrated that the Herschel-SPIRE data are indeed able to discriminate between the two emission mechanisms and therefore detect the effects of the AGN (van der Werf et al. 2010). Herschel-PACS observations of the high-J CO lines $\left(\mathrm{J}_{u p} \geq 14\right)$ detected in NGC 1068 have been recently presented by Hailey-Dunsheath et al. (2012). The two components, at high and medium excitation, needed to explain the observed CO lines arising from the central $10^{\prime \prime}$ region from $\mathrm{J}_{u p}=14$ to $\mathrm{J}_{u p}=24$ can be excited by $\mathrm{X}$-ray or shock heating, while far-UV heating is unlikely.

The Herschel-SPIRE spectroscopic observations of NGC 1068 presented here have been collected under the guaranteed time key project "Physical Processes in the Interstellar Medium of Very Nearby Galaxies" (PI: Christine Wilson). Within the same observational program, SPIRE and Photodetector Array Camera and Spectrometer (PACS) (Poglitsch et al. 2010) photometric images have been collected, which will be presented in a forth- coming paper with the detailed analysis of the continuum emission (Spinoglio et al 2012, in prep.).

\section{OBSERVATIONS}

\subsection{SPIRE Spectroscopy}

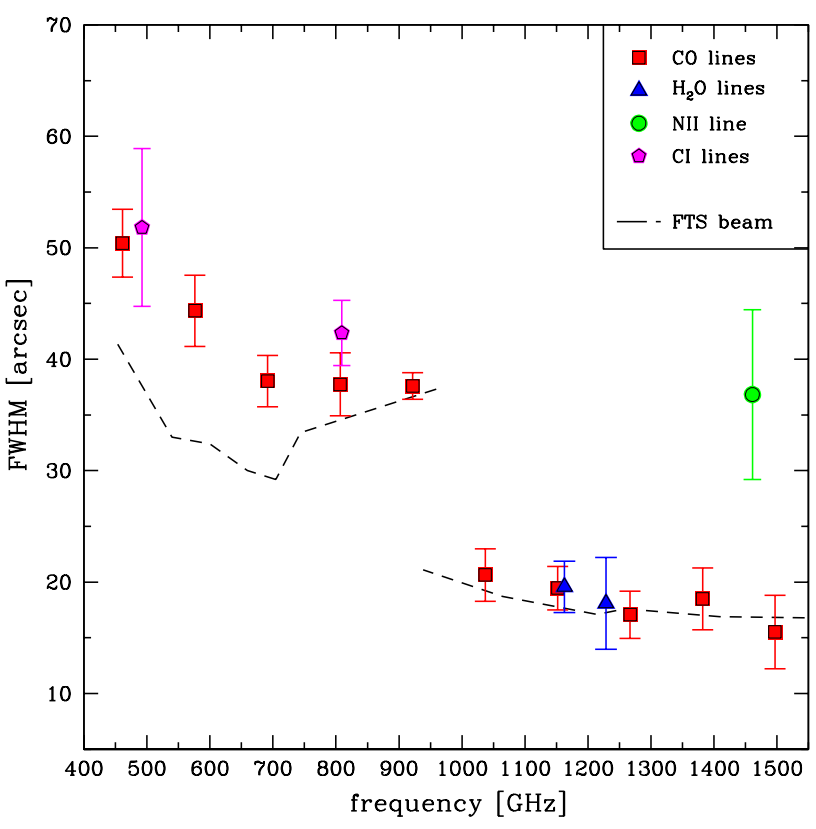

FIG. 1.- Observed source sizes, as measured from the mapping observations of the brightest SPIRE lines (FWHM in arcsec, with $1 \sigma$ error bars) as a function of frequency (colored points) compared with the SPIRE-FTS beam (broken lines). All source sizes in the SSW, except for $[\mathrm{NII}] 205 \mu \mathrm{m}$, are consistent with the beam width at that frequency. In contrast, the source sizes in the SLW are more extended than the beam.

NGC 1068 was observed with the SPIRE-FTS (Griffin et al. 2010) onboard the Herschel Space Observatory (Pilbratt et al. 2010) both in the single pointing mode with sparse image sampling and in the mapping mode with full image sampling. The FTS has two detectors arrays called the spectrometer long wave (SLW, in the range of 303-671 $\mu \mathrm{m}$ ) and the spectrometer short wave (SSW, in the range $194-313 \mu \mathrm{m})$, with a small (10 $\mu \mathrm{m})$ overlap in wavelength. The spectral resolution of the FTS ranges from about $280 \mathrm{~km} \mathrm{~s}^{-1}$ to $950 \mathrm{~km} \mathrm{~s}^{-1}$ in the high resolution mode, moving in wavelength from $194 \mu \mathrm{m}$ to 671 $\mu \mathrm{m}$. The two SPIRE FTS observations of NGC 1068 were collected on Operational Day 626 (29 January 2011); the fully sampled map has Observation ID 1342213444 and the single pointing "deep" spectrum has Observation ID 1342213445. The pointed observations were carried out both at low and high spectral resolutions $(\mathrm{FWHM} \sim 30$ $\mathrm{GHz}$ and $1.44 \mathrm{GHz}$ ), covered with 32 repetitions each, for a total on-source integration time of 410 seconds and 4,262 seconds respectively ${ }^{10}$. The shallower mapping observations at high resolution had 8 repetitions and covered an area with a diameter of approximately $2^{\prime}$. The total on-source integration time was 17,050 seconds.

The FTS beam size and shape vary as a function of frequency and cannot be characterized by a simple Gaussian

10 See the Herschel SPIRE Observers manual, available at http://herschel.esac.esa.int/Docs/SPIRE/html/spire_om.html 

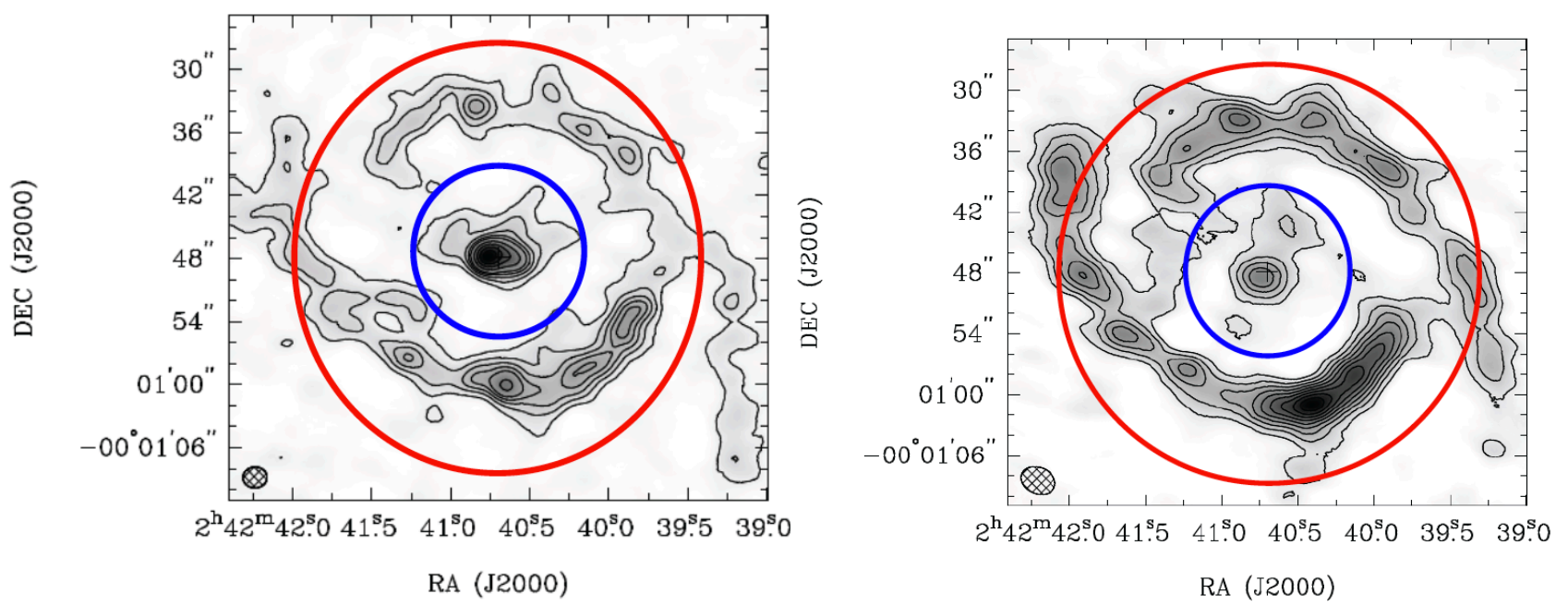

FIG. 2.- Left: $\mathrm{CO}(3-2)$ integrated intensity map of the central region of NGC 1068 from Tsai et al. (2012). Right: CO(1-0) integrated intensity map of the central region of NGC 1068 from Tsai et al. (2012). The minimum SSW (blue) and the maximum SLW (red) SPIRE FTS beam sizes of $17^{\prime \prime}$ and $42^{\prime \prime}$, respectively, are shown in the figures as concentric circles.

response. As can be seen from Figure 1, where the observed source sizes of the brightest lines detected by the mapping observations of the SPIRE FTS are plotted as a function of frequency (see the method described in Section 2.1.1), the FTS beam widths (FWHM) range from $17^{\prime \prime}$ to $42^{\prime \prime}$, probing a wider area in the sky with increasing wavelength, and show a strong discontinuity between the SLW and SSW bands, due to the multi-moded feedhorns used for the spectrometer arrays (see Section 4.2.3 and Figure 5.13 in the SPIRE Observers' Manua ${ }^{10}$.

The combination of the complex morphology of the inner region of NGC 1068 with the SPIRE-FTS beam properties yield a particular coupling effect between the two. Figure 2 shows the $\mathrm{CO}(3-2)$ and $\mathrm{CO}(1-0)$ interferometric maps from Tsai et al. (2012) of the central region of NGC 1068. As can be seen from these maps, the morphology of NGC 1068 is dominated by two main components (see also, e.g., García-Burillo et al. 2010, and references therein): the compact (of the order of $4^{\prime \prime}$ in diameter) CND at the center of the galaxy, possibly associated with the active nucleus, and a relatively extended ring, with a radius of the order of 10-20", whose emission is dominated by star formation (SF) activity. The relative contribution from these two spatial components to the various rotational transitions of the ${ }^{12} \mathrm{CO}$ molecule strongly varies with the transition from the low$\mathrm{J}\left(\mathrm{J}_{u p} \sim 4\right)$ to the high-J $\left(\mathrm{J}_{u p} \sim 13\right)$ lines. In this range the dominant source of $\mathrm{CO}$ excitation changes from the extended SF-ring to the compact CND. There is also a variation of the angular scale probed by the FTS beam, from extended (beam FWHM $\sim 42^{\prime \prime}$ ) to compact $\left(17^{\prime \prime}\right)$, as shown by the two concentric circles in the maps of Figure 2

\subsubsection{Source size measurements}

We first describe the reduction procedure that was used for source size measurements, while in Sections 2.1.2 and 2.1 .3 we describe the reduction procedure for spectral line measurements. We employ two reductions as the two measurements have different requirements.

The mapping observations of NGC 1068 have been reduced with the standard reprocessing pipeline in HIPE

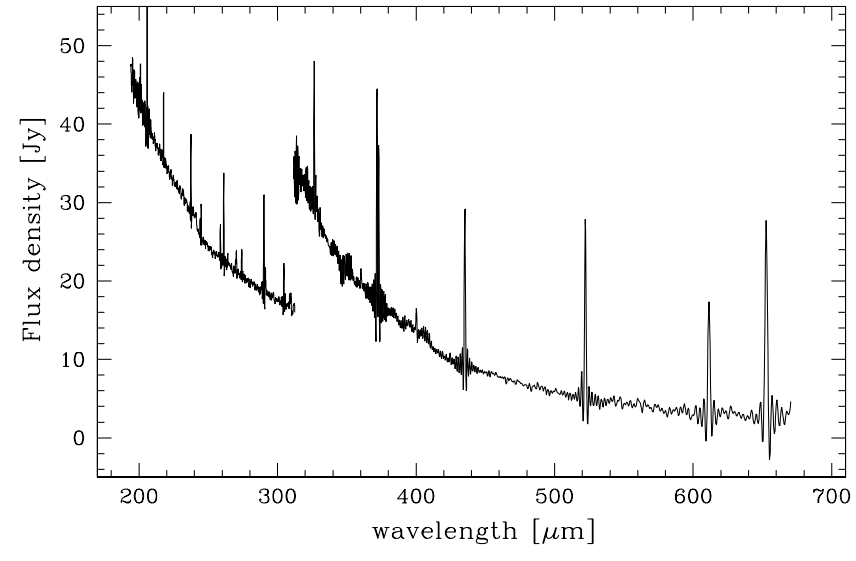

FIG. 3.- The observed submillimeter spectrum of NGC 1068, as measured from the SPIRE-FTS pointed observations. As can be seen the two SSW and SLW spectra do not overlap. This is due to both the different beam sizes and the intrinsically different source sizes.

v.7 and converted into spectral cubes using the NAIVEProjeCtion task ${ }^{11}$ with custom pixel sizes of $11^{\prime \prime}$ and $13.8^{\prime \prime}$ for the SSW and SLW bands, respectively. These sizes were chosen empirically starting from a large size and reducing it until holes (pixel with zero coverage) appeared in the maps. The final cube for each of the two FTS bands has a variable on-source integration time in the map, between 16 and 32 FTS scans. From these two cubes a number of sub-cubes have been obtained by extracting a $60 \mathrm{GHz}$ wide window (corresponding to $\sim 200$ bins in high spectral resolution) around each line detected in the spectrum of the brightest spaxel, where a spaxel is defined as each one of the $5 \times 5$ spatial pixels which fill the spectrometer field of view ${ }^{12}$ and modeling and subtracting the continuum emission with a $1^{\text {st }}$ order

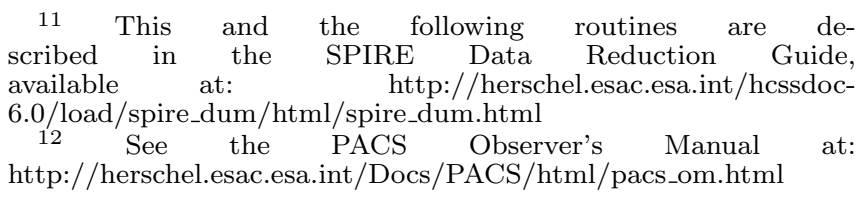




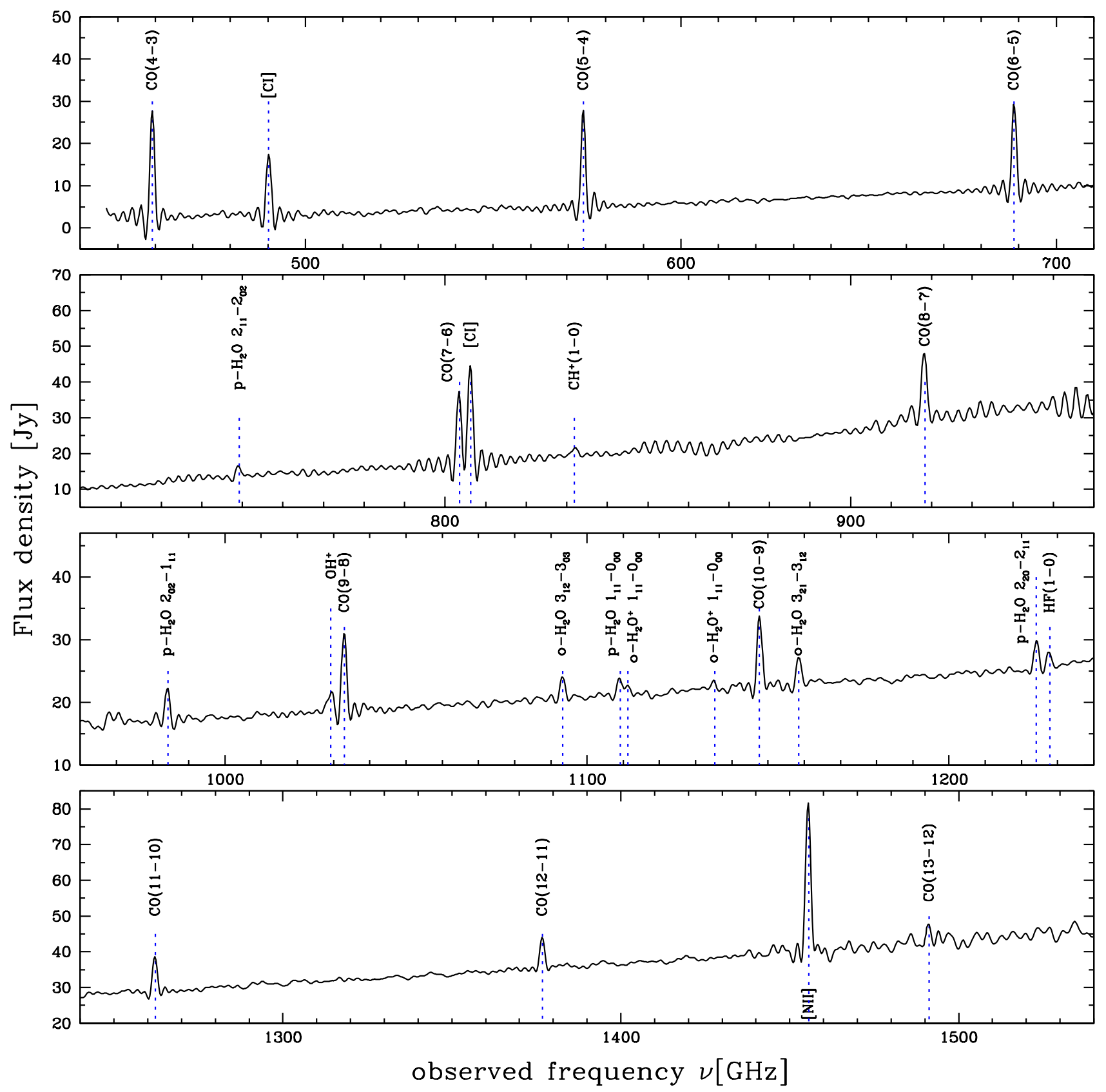

FIG. 4. - The observed submillimetre spectrum of NGC 1068, as measured from the SPIRE-FTS pointed observations. From top to bottom are presented the consecutive spectral ranges. The emission line positions are indicated. Line identifications are given for detections with $\mathrm{S} / \mathrm{N}>3 \sigma$ (see Table 1).

polynomial using HIPE's task LinEINTENSITYMAP.

The FTS data from a pointed observation have two available calibrations which provide the target's correct flux for the two extreme cases of an unresolved source and of an extended source that completely and uniformly fills the instrument beam. To determine which calibration to use, we measured the emitting region size for several lines from the analysis of the spectra cubes (see Figure 1).

The source sizes of all the transitions present in the SSW band, with the exception of the $[\mathrm{NII}] 205 \mu \mathrm{m}$ line, are consistent with originating from an unresolved source and they are therefore well calibrated in the pointed spec- trum using the unresolved source solution. For this reason, we used the line fluxes measured from the pointed spectrum for all the transitions in the SSW band except for the $[\mathrm{NII}] 205 \mu \mathrm{m}$ line, for which we have used the mapping observations. ([NII] emission arises from ionized gas and so we may expect some contribution from the SF-ring.) For all the transitions in the SLW band, we measured the source sizes from the mapping observations.

We have obtained line integrated maps with the task INTEGRATEMAPFROMCUBE from the spectral subcubes. The integration over the lines has made no use of 
the fitting functionality inside the task, because of the possible spurious detections observed for spectral features with low signal-to-noise ratios. These line maps have been used to measure the angular extent of the region emitting every detected line using a 2D Gaussian fit. While the instrument PSF is not strictly gaussian, because a large fraction of its light is in wider secondary lobes, this approach is robust enough to quantify the scale size of the emitting region through the fit of the PSF core.

In conclusion, the deep pointed spectrum in the SSW band samples primarily, not exclusively, the lines emitted by the compact source, while the SLW band measures lines emitted by both the compact and the extended structures. This coupling effect between the beam and the target morphology makes it difficult to reconstruct the correct spectral line energy distribution (SLED) for the ${ }^{12} \mathrm{CO}$ molecular lines for the CND alone. To overcome these problems, we have combined the information extracted from the pointed spectrum with those derived from the mapping observations.

\subsubsection{Spectroscopic mapping observations}

The mapping observations of NGC 1068 were again reduced using a modified version of the standard Spectrometer Map pipeline script in the Herschel Interactive Processing Environment (HIPE) v.9, developers build 588 and version 8.1 of the SPIRE calibration context. The standard reduction assumes that the source is extended and uniformly fills the beam. Since our source does not fill the beam, the unique point source flux conversion measured for each bolometers was applied to each of the bolometers in our two arrays. After the point source correction, spectral cubes for the SSW and SLW were created using the spireProjection task with projectionType="naive" with a custom pixel size of $11^{\prime \prime}$ and $13.8^{\prime \prime}$ respectively as before. This cube was used for all SLW and the $[\mathrm{NII}] 205 \mu \mathrm{m}$ line intensity measurements.

\subsubsection{Pointed observations}

The single pointing/sparse observations of NGC 1068 have been reduced using the Spectrometer Single Pointing user pipeline in the Herschel Interactive Processing Environment (HIPE) v.9, developers build 588 and version 8.1 of the SPIRE calibration context. An older version of this pipeline is described in Fulton et al. (2010). The observed submillimeter spectrum of NGC 1068, as measured from the SPIRE-FTS pointed observations, is shown in Figure 3, where the large offset at $\sim 300 \mu \mathrm{m}$ is due to the large difference in the beam of the SSW and the SLW spectrometers.

\subsection{Line intensities}

As explained in the previous section, the transitions measured in the SSW band, except for the $[\mathrm{NII}] 205 \mu \mathrm{m}$ line, have been considered spatially unresolved and therefore measured from the pointed/deep spectrum, while, on the contrary, the other partially extended lines have been measured from the mapping observations.

In order to measure the line fluxes of the extended component, the line maps have been convolved with $2 \mathrm{D}$ kernels (Bendo et al. 2012) to reach the same beam size at every frequency. The final PSF corresponds to the coarsest angular resolution $\left(\mathrm{FWHM}=42^{\prime \prime}\right)$ reached by the FTS for a ${ }^{12} \mathrm{CO}$ line (i.e. the $\mathrm{CO}$ (4-3) at 461.04 $\mathrm{GHz}$ ). The kernels were built using the SPIRE-FTS PSF measurements, dividing the PSF image of the CO (4-3) line by that of each detected line in the Fourier space.

The baseline was removed by first masking all of the spectral lines before fitting a high order polynomial to the remaining continuum. The intensities of the detected lines have been computed with a Levenberg Marquardt fitting procedure using the sinc function model. Table 1 presents, for each detected transition, the rest frequency, the energy of the upper level (in K), the beam FWHM (in arcsec.)(see the SPIRE Observers' Manual10 the intensity value with both the $1 \sigma$ statistical uncertainty and total uncertainty, which include the calibration uncertainty added in quadrature. For the mapping observations, we have adopted a fixed FWHM of $43.4^{\prime \prime}$ for all detected lines. For the pointed observations of the compact source, we have adopted a calibration uncertainty of $10 \%$, while for the mapping observations of the extended source, in addition to the calibration uncertainty, we have also added in quadrature a flat-fielding uncertainty of $7 \%$ (see the SPIRE Observers' Manua ${ }^{10}$. The lines detected with the FTS are not spectrally resolved.

Figure 4 presents the observed spectrum of NGC 1068 with the identifications of all lines detected at $\mathrm{S} / \mathrm{N}>3$. A total of 24 emission emission lines have been detected with the Herschel-SPIRE FTS: ten lines from CO (from $\mathrm{J}_{\text {up }}=4$ to $\mathrm{J}_{\mathrm{up}}=13$ ), six lines from $\mathrm{H}_{2} \mathrm{O}$, two from $\mathrm{H}_{2} \mathrm{O}^{+}$, one from $\mathrm{OH}^{+}, \mathrm{HF}$ and $\mathrm{CH}^{+}$each, in addition to the three atomic lines (from $[\mathrm{NII}]$ and $[\mathrm{CI}]$ ). The apparent ringing in the spectrum is due to the sinc response of the FTS to each line.

In Table 1 we also included literature measurements, relative to both the compact source and the extended source. In particular, we considered in our analysis the interferometric observations of CO (1-0), (2-1) and (32) from Krips et al. (2011), and the water lines from the Herschel-PACS observations (S. Hailey-Dunsheath 2012, private communication) for the compact component, and, for the extended component, CO (2-1) Z-Spec spectrometer observations at the Caltech Submillimeter Observatory (CSO) (Kamenetzky et al.2011) and James Clerk Maxwell Telescope (JCMT) CO (3-2) observations convolved to the $43.4^{\prime \prime}$ beam (C. Wilson 2012, private comm.).

\section{RESULTS AND DISCUSSION}

\subsection{CO Radiation transfer modeling}

To obtain a general solution for the CO physical conditions, we have first used the radiative transfer code RADEX (van der Tak et al. 2007). Then, considering the best possible ranges of the parameters as derived from RADEX, we have also used the PDR and XDR models from Wolfire et al. (2010) and Meijerink \& Spaans (2005) and Meijerink et al. (2007), respectively, to provide constraints on the origin of the excitation of the measured lines, and in particular to determine whether there is an influence of the AGN, through its X-ray emission, on the molecular gas in the CND and SF-ring associated with NGC 1068.

\subsubsection{RADEX models}


RADEX is a non-Local Thermodynamic Equilibrium (LTE) code available from the Leiden Atomic and Molecular Database (LAMDA, Schöier et al. 2005). Under the assumption of a uniform medium, using an escape probability formalism that models the entire emitting region as a single zone, RADEX performs statistical equilibrium calculations involving collisional and radiative processes. We used the collisional rate coefficients with $\mathrm{H}_{2}$ of Yang et al. (2010). Considering the radiation field from background sources given as input, it computes the molecular level populations in the optically thin limit in the first iteration and the optical depths and the escape probabilities for each line. The interdependence of the molecular level populations and the local radiation field requires that the solution is reached through an iterative method. At each iteration, the code computes new level populations using new optical depth values, until the two converge on a consistent solution. Then the resulting line intensities are given as output.

The input parameters required for RADEX are the background radiation field, the kinetic temperature $\mathrm{T}_{k i n}$, the molecular hydrogen number density $\mathrm{n}\left(\mathrm{H}_{2}\right)$ (assumed to be the only collision partner), and the ratio between the column density of the molecule and the width of the lines, which is the physically relevant quantity that determines the optical depth.

We used RADEX to create a grid of solutions for the $\mathrm{CO}$ transitions spanning wide ranges in $\mathrm{T}_{k i n}, \mathrm{n}\left(\mathrm{H}_{2}\right)$, and $\mathrm{N}_{C O}$ (see Table 2). To compare this grid of models with our CO measurements we used the Bayesian likelihood analysis code (Ward et al. 2003), developed by the Z-Spec Team (Naylor et al. 2010a; Kamenetzky et al. 2011).

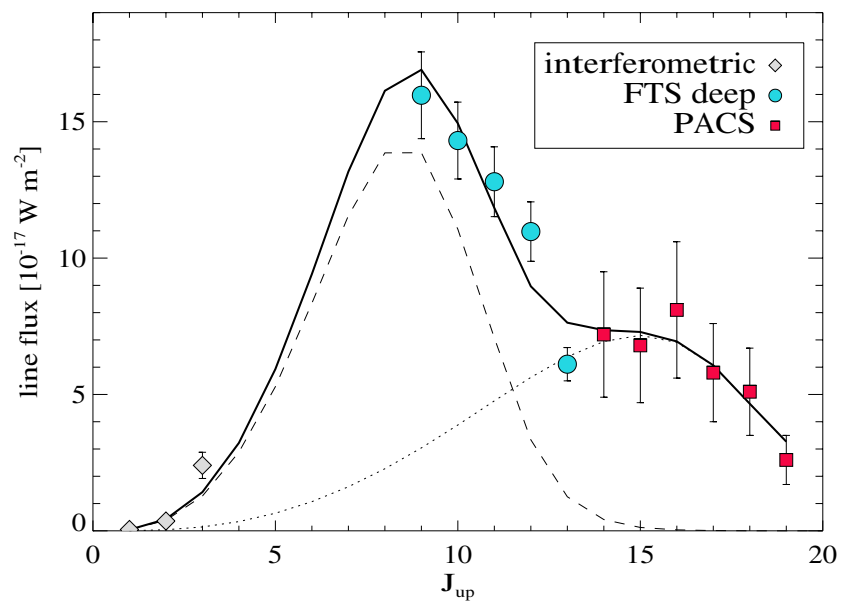

FIG. 5.- RADEX model fitting for the compact component associated with the CND. Square points (red) represent the PACS data, circle points (blue) depict the FTS deep spectrum data, and the diamond points (grey) show the interferometric observations of the CND (Krips et al. 2011), the dotted line shows the ME model of Hailey-Dunsheath et al. (2012), while the dashed line shows the best fitting configuration of RADEX parameters (see column 4D Max of Table 3. The sum of the two models is shown as a solid line.

Computing the RADEX models, we have considered both models with and without the inclusion of the local background radiation to be added to the cosmological component of the CMB at $2.73 \mathrm{~K}$. To estimate the local background in the far-IR, we have fitted the aperture photometry of the Herschel-PACS and SPIRE

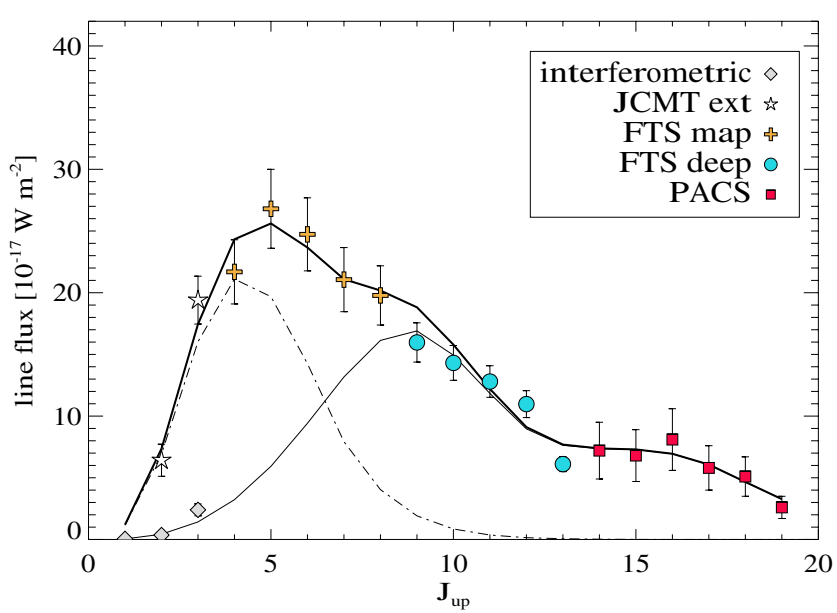

FIG. 6. - RADEX model fitting for the extended component associated with the SF-ring. Notations as in Figure 5 The crosses (yellow) represent the FTS measurements from the spectral map and the white stars show the ground-based observations of the extended component (Kamenetzky et al. 2011, Wilson 2012, private communication). The dot-dashed line shows the best fitting configuration of RADEX parameters (see column 4D Max of Table 3 . The thin solid line is the best model for the CND component. The sum of all models is shown as a thick solid line.

maps at 70, 160, 250, 350 and $500 \mu \mathrm{m}$, respectively, that will be presented and discussed in a forthcoming paper (Spinoglio et al. 2012, in prep.). We have used a circular aperture with a diameter of $33^{\prime \prime}$ and a dust emissivity law with $\beta=2$ and we have derived a gray-body temperature of $35 \mathrm{~K}$. In agreement with the higher J-CO line fitting results of Hailey-Dunsheath et al. (2012), we have found that the inclusion of this local background does not change significantly the results of the CO fits that only include the CMB. Therefore we have adopted only the $\mathrm{CMB}$ black-body radiation at $2.73 \mathrm{~K}$ as background radiation, for both the extended and compact sources.

The presence of several spatial and spectral components in the CO SLED has required a multi-step approach, looking for the best solution of one component at a time and subtracting it from the others. We started by considering the CO SLED of the compact source, composed of the FTS-SSW measurements in combination with the compact interferometric observations. For the high-J $\left(J_{\text {up }} \geq 14\right)$ PACS data, we used the medium excitation (ME) component LVG model presented by HaileyDunsheath et al. (2012), characterized by a density of $10^{5.7} \mathrm{~cm}^{-3}$ and a kinetic temperature $T_{\text {kin }}=150 \mathrm{~K}$. The $\mathrm{ME}$ model for the $\mathrm{J}_{\mathrm{up}}>13 \mathrm{CO}$ lines was subtracted from the observed CND spectrum before attempting to fit the SLED.

In Table 3 the results obtained with the Z-Spec code are summarized. For each of the two models of the CND and SF-ring the following parameters are given: the kinetic temperature of the CO, $T_{\text {kin }}$, the molecular hydrogen density, $n\left(\mathrm{H}_{2}\right)$, the $\mathrm{CO}$ column density, $N_{C O}$, the area filling factor, $\Phi_{\mathrm{A}}$, the gas pressure, $P$, the beam averaged $\mathrm{CO}$ column density, $\left\langle N_{\mathrm{CO}}\right\rangle$, and the molecular hydrogen mass $\mathrm{M}\left(\mathrm{H}_{2}\right)$, that has been computed using a CO abundance of $x_{C O}=3 \times 10^{-4}$, adopted by the Z-Spec code (Naylor et al. 2010a, Kamenetzky et al. 2011).

For each parameter, the median value, the 1- $\sigma$ interval, the $1 \mathrm{D}$ and the $4 \mathrm{D}$ maximum values are given. $1 \mathrm{D}$ refers to the maximum value of the integrated parameter dis- 
tribution while $4 \mathrm{D}$ refers to the value of that parameter at the best fit solution.

The compact source is unresolved with all the considered instruments and identified with the CND, while the extended source data probe the emission arising from a region of $43.4^{\prime \prime}$ in diameter, compatible with the SF-ring structure. We have obtained the best fit to the CND with RADEX, as can be seen in Figure 5, by fitting the mid-J lines (from $\mathrm{J}_{u p}=9$ to $\mathrm{J}_{u p}=13$ ) together with the low$\mathrm{J}$ lines observed with interferometric techniques, which isolate the CND component, and subtracting the contribution from the medium excitation (ME) component of the LVG model of Hailey-Dunsheath et al. (2012).

The best fit of the SF-ring with RADEX (see Figure 6) was then obtained by subtracting from the observed lines (from $\mathrm{J}_{u p}=4$ to $\mathrm{J}_{u p}=8$ with SPIRE and $\mathrm{J}_{u p}=3$ and 2 with JCMT) the contribution arising from the compact source by summing the mid-J and high-J contributions. As can be seen in Figure 6, the extended source gives a significant contribution to the over-all SLED only for $\mathrm{J}_{u p}<9$. To summarize, as can be seen in Table 3, we found that the best fit RADEX model results for the CND give $T_{\text {kin }}=100 \mathrm{~K}$ and $n\left(\mathrm{H}_{2}\right)=10^{4.5} \mathrm{~cm}^{-3}$ and for the SF-ring $T_{\text {kin }}=127 \mathrm{~K}$ and $n\left(\mathrm{H}_{2}\right)=10^{2.9} \mathrm{~cm}^{-3}$.

\subsubsection{PDR and XDR models of the CND component}

PDR and XDR models from Wolfire et al. (2010) and from Meijerink \& Spaans (2005) and Meijerink et al. (2007) 13 describe the thermal and chemical balance of molecular gas that is exposed to far-ultraviolet (FUV) radiation (6-13.6 eV) and X-rays (1-100 keV), respectively. The codes used to determine the gas conditions in these regions as a function of depth take into consideration elaborate chemical networks and the cooling, heating and chemical processes which are completely determined by the radiation field.

We have considered the grids of PDR models of Wolfire et al. (2010), that span from $10^{1}$ to $10^{7} \mathrm{~cm}^{-3}$ in density and from $10^{-0.5}$ to $10^{6.5} \mathrm{G}^{14}$ in FUV flux. The PDR models are based on those of Kaufman et al. (2006), with updates from Wolfire et al. (2010) and Hollenbach et al. (2012). In particular the atomic and molecular freeze-out and grain chemistry in Hollenbach et al. (2012) are included, as well as their PAH photo rates which affect the ion-neutral chemistry and the production of CO. We use their "linear yield" $\mathrm{PAH}^{0}$ photoionization rate slightly modified for a Draine $(1978)$ interstellar radiation field. Hot $\mathrm{CO}$ production by $\mathrm{HCO}^{+}$recombinations is also included, as suggested by J. Black (2012, private communication).

For the XDR models, we used the grid by Meijerink et al. (2007) that spans two different ranges in density $\left(10^{3}-10^{4} \mathrm{~cm}^{-3}\right.$ and $\left.10^{4}-10^{6.5} \mathrm{~cm}^{-3}\right)$ irradiation $\left(F_{X}=\right.$ $\left.1.6-160 \mathrm{ergs}^{-1} \mathrm{~cm}^{-2}\right)$, and cloud size of $1 \mathrm{pc}$ and $10 \mathrm{pc}$. We used a $\chi^{2}$ minimization algorithm to identify the best description of the ${ }^{12} \mathrm{CO}$ line fluxes measured with SPIRE and interferometric data (see Table 1).

As already mentioned in Section 3.1.1, in addition to the CO lines detected in NGC 1068 by the SPIRE FTS,

\footnotetext{
13 Available at: http://www.strw.leidenuniv.nl/meijerink/grid/

${ }^{14} \mathrm{G}_{0}=1.6 \times 10^{-3} \mathrm{erg} \mathrm{cm}^{-2} \mathrm{~s}^{-1}$ and the field is integrated from 6 to $13.6 \mathrm{eV}$.
}

presented in Table 1 , higher $\mathrm{J}$ lines (from $\mathrm{J}_{u p}=14$ to $\mathrm{J}_{u p}=24$ ) have been observed by PACS and presented in Hailey-Dunsheath et al. (2012). We have tried to model the whole sequence of CO lines detected with PDR/XDR models, however no single component was able to fit the data; three distinct components were needed, all originating from the compact CND, to fit the CO lines down to $\mathrm{J}_{u p}=9$. Moreover, as outlined in Hailey-Dunsheath et al. (2012), even if - in principle - PDR models could fit the data from $\mathrm{J}_{u p}=14$ to $\mathrm{J}_{u p}=24$, the morphology of the $\mathrm{H}_{2}$ near-IR emission and the two different kinematics of the $\mathrm{CO}$ lines at $\mathrm{J}_{u p} \leq 17$ and $\mathrm{J}_{u p} \geq 20$, indicate that these two sets of lines trace physically distinct components, therefore excluding a PDR origin of these high-J $\mathrm{CO}$ lines. Consequently, for the high-J $\left(\mathrm{J}_{u p} \geq 14\right)$ PACS data we used the model presented by Hailey-Dunsheath et al. (2012), consisting of two XDR components with a density of $10^{5.75} \mathrm{~cm}^{-3}$ and incident X-ray fluxes of 9 and $160 \mathrm{erg} \mathrm{s}^{-1} \mathrm{~cm}^{-2}$, respectively. Then we have performed the analysis on both the compact source and the extended source, once the other components were fitted and removed as explained in the previous section.

The mid-J lines originating from the CND are best described by a sequence of PDR models ranging from an incident FUV flux of $\mathrm{G}_{o} \sim 10^{5.0}$ and density of $10^{4.5} \mathrm{~cm}^{-3}$, in agreement with the RADEX model, to a FUV flux of $\mathrm{G}_{o} \sim 10^{2.0}$ and density of $\sim 10^{6.0} \mathrm{~cm}^{-3}$ (see left panel of Figure 7). On the other hand, also XDR models either with density $\sim 10^{4.0} \mathrm{~cm}^{-3}$ or $\sim 10^{6.0} \mathrm{~cm}^{-3}$ and incident $\mathrm{X}$-ray flux in the range of $1.6-5.0 \mathrm{erg} \mathrm{s}^{-1} \mathrm{~cm}^{-2}$ are able to reproduce the observed mid-J CO fluxes with a good $\chi^{2}$ value (see right panel of Figure 7). However, the RADEX models (see section 3.1.1) favor solutions with $\mathrm{n}_{H}$ between $10^{4.4}$ and $10^{4.8} \mathrm{~cm}^{-3}$ as listed in Table 3 .

To further constrain the models, we have used the $\mathrm{CO}$ surface brightness for both PDR and XDR models, by summing over all of the modeled CO lines. For the CND, we have computed the total $\mathrm{CO}$ emission from the best fit XDR model presented in Figure 10 and divided by the emitting area of the CND, assumed to be 4 " in diameter, and corrected by the filling factor of the RADEX best fit (see Table 3) to derive the CO surface brightness of the CND.

In Figures 8 and 9 we present the total CO surface brightness for both PDR and XDR models, respectively, with the density region allowed by the RADEX models indicated by shaded regions. From Figure 8 , the predicted $\mathrm{CO}$ surface brightness in the CND by PDR models, which satisfies the density constrain of the RADEX model, is about two orders of magnitude below the value computed with the best fit model of the CND of $0.02 \mathrm{erg} \mathrm{s}^{-1} \mathrm{~cm}^{-2} \mathrm{sr}^{-1}$. On the other hand, Figure 9 shows that the predicted CO surface brightness in the CND, for an XDR model with density of $\sim 10^{4.3} \mathrm{~cm}^{-3}$ and incident X-ray flux $\geq 3 \mathrm{erg} \mathrm{s}^{-1} \mathrm{~cm}^{-2}$, agrees well with the best fit value of $0.02 \mathrm{erg} \mathrm{s}^{-1} \mathrm{~cm}^{-2} \mathrm{sr}^{-1}$.

We have also compared the observed parameters of the CND in NGC 1068 with the XDR model results presented for Arp 220 in Rangwala et al. (2011). The XDR model of Arp 220 has been computed using an updated version of the code described by Maloney et al. (1996). The assumed physical conditions in Arp 220 coincide with those of NGC 1068: a hard X-ray (1-100keV) luminosity of $\mathrm{L}_{X}$ 

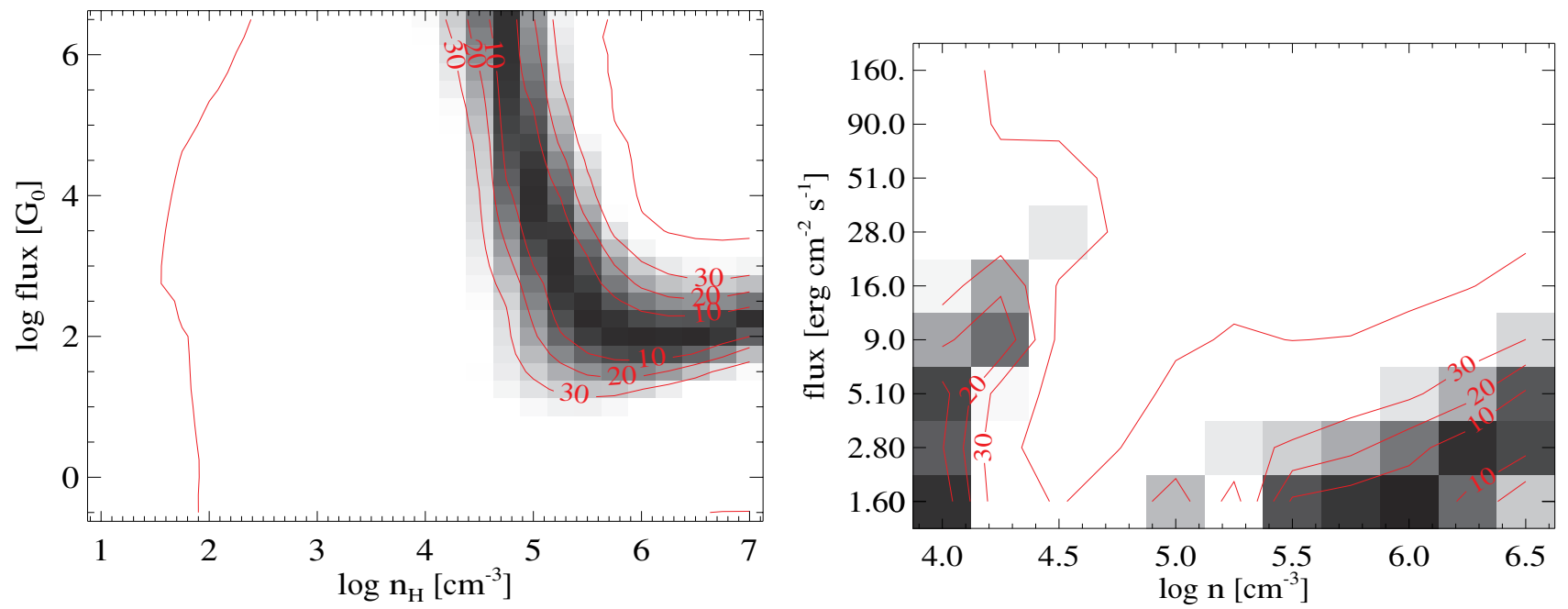

FIG. 7.- Reduced $\chi^{2}$ values of the models for the CND. Left: PDR models (Wolfire et al. 2010) ; Right: XDR models (Meijerink \& Spaans 2005 Meijerink et al. 2007).

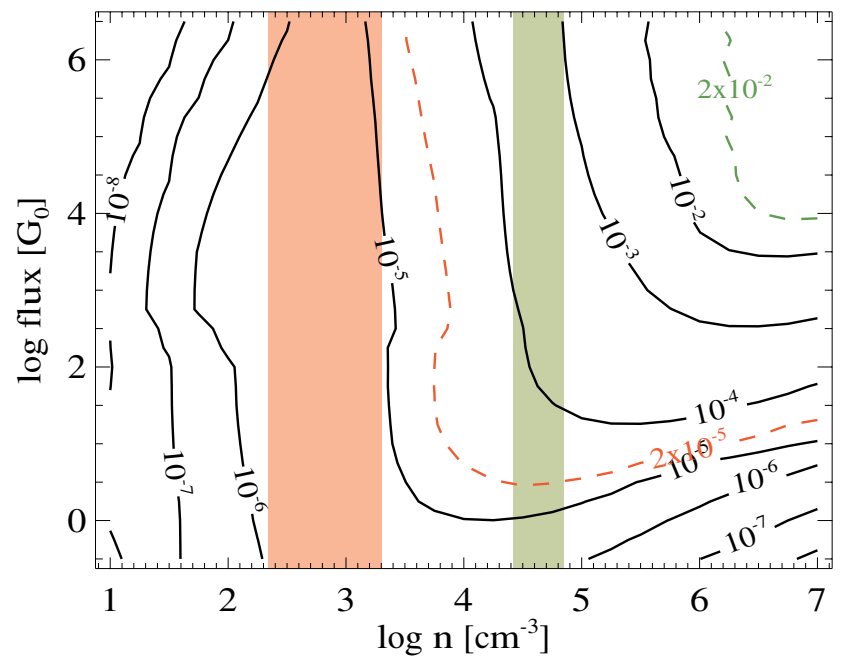

FIG. 8.- Total CO surface brightness (in units of erg s${ }^{-1} \mathrm{~cm}^{-2} \mathrm{sr}^{-1}$ ) as a function of incident FUV flux and density for the PDR models. The left shaded region (in orange) shows the $1 \sigma$ range of the density of the best fit RADEX model of the SF-ring (extended region), while the right shaded region (in green) reports the area of the $1 \sigma$ range of the density of the best fit RADEX model of the CND (compact component). The red dashed line represents the measured surface density for the SF-ring, while the green dashed line represents the measured surface density for the CND of $0.02 \mathrm{erg} \mathrm{s}^{-1} \mathrm{~cm}^{-2} \mathrm{sr}^{-1}$.

$\sim 10^{44} \mathrm{erg} \mathrm{s}^{-1}$, a power law index of $\alpha=0.7$ and an absorbing hydrogen column density of $\mathrm{N}_{H}=10^{24} \mathrm{~cm}^{-2}$, typical of Compton thick sources, like NGC 1068. In their Figure 7 (bottom, right panel), Rangwala et al. (2011) show the predicted CO surface brightness as a function of density and radius of the emitting region. Our best fit value of $0.02 \mathrm{erg} \mathrm{s}^{-1} \mathrm{~cm}^{-2} \mathrm{sr}^{-1}$ is in reasonable agreement with a density of $\sim 10^{4} \mathrm{~cm}^{-3}$ and a radius of $100-200 \mathrm{pc}$. The radius that we associate to the $\mathrm{CO}$ emitting region in the CND is about $160 \mathrm{pc}$. We therefore conclude that the XDR origin of the CO CND emission in NGC 1068 is indeed a plausible explanation. In Figure 10 we show the fit to the observed $\mathrm{CO}$ fluxes using the best fit XDR model with the adopted density and X-ray flux for the CND component.

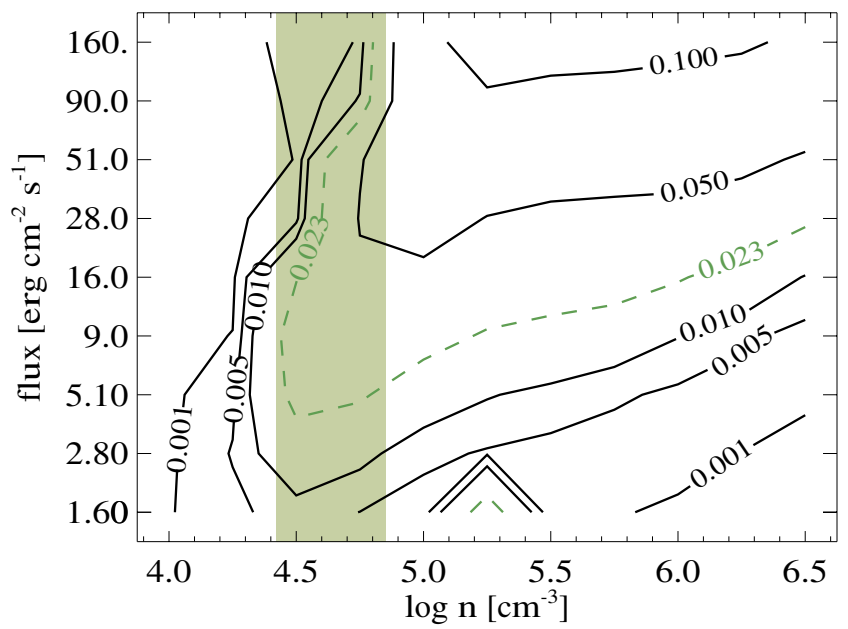

FIG. 9.- Total CO surface brightness (in units of $\mathrm{erg} \mathrm{s}^{-1} \mathrm{~cm}^{-2} \mathrm{sr}^{-1}$ ) as a function of incident FUV flux and density for the XDR models. The shaded region (in green) shows the $1 \sigma$ range of the density of the best fit RADEX model of the CND (compact component). The dashed line (in green) represents the measured surface density.

\subsection{3. $P D R$ and XDR models of the SF-ring component}

The low-J component of the SLED can be fitted either by PDR models with a FUV flux of $\mathrm{G}_{o} \gtrsim 10^{2}$ and density of $\sim 10^{3.5} \mathrm{~cm}^{-3}$, in rough agreement with the RADEX modeling results, or with models with lower FUV flux $\left(\mathrm{G}_{o} \sim 10^{1}\right)$ and a higher density of $\gtrsim 10^{4} \mathrm{~cm}^{-3}$, which are, however, in conflict with the RADEX results. Moreover (see the right of Figure 11), XDR models with a density $\sim 10^{3.0} \mathrm{~cm}^{-3}$ and incident $\mathrm{X}$-ray flux in the range of $0.5-$ $2.0 \mathrm{erg} \mathrm{s}^{-1} \mathrm{~cm}^{-2}$ can fit the data.

Similarly to the case of the CND, described in Section 3.1.2 for the SF-ring we have used the total CO emission from the best fit PDR model and the area of $40^{\prime \prime}$ in diameter to derive the extended component surface brightness. For this latter case, we did not use any filling factor because we consider that the extended emission in the SF-ring is filling most of the beam.

To further constrain the models, we use again the $\mathrm{CO}$ surface brightness. From Figure 8, the predicted CO surface brightness in the SF-ring, for a PDR model with 


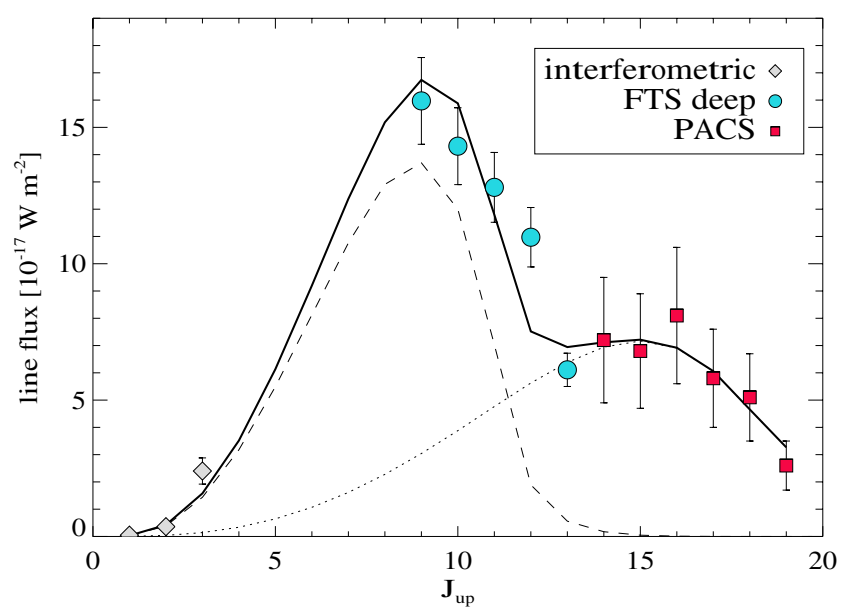

FIG. 10. - XDR model fitting for the compact component, associated with the CND. Models have been taken from Meijerink $\&$ Spaans (2005) and Meijerink et al. (2007). The points follow the same color code as in Figure 5 . The dotted line shows the fit of the high-J CO lines with the XDR model of Hailey-Dunsheath et al. (2012), the dashed lines show the fit of the intermediate-J CO lines, with a XDR model with density $n=10^{4.25} \mathrm{~cm}^{-3}$ and X-ray flux of $9 \mathrm{erg} \mathrm{s}^{-1} \mathrm{~cm}^{-2}$, once the high-J CO XDR model has been subtracted. The solid line shows the total modeled emission, which is compared to the observed data (see the text).

density of $\sim 10^{3} \mathrm{~cm}^{-3}$ and incident FUV flux above $G_{0} \geq$ $10^{2}$, agrees well with the observed value of $2 \times 10^{-5}$ erg $\mathrm{s}^{-1} \mathrm{~cm}^{-2} \mathrm{sr}^{-1}$, reinforcing this solution. We present the best fit model in Figure 12 .

\subsubsection{Constraining the PDR and XDR models with luminosities}

In order to verify that the XDR models used to fit the compact (CND) and extended (SF-ring) molecular components in NGC 1068 are in agreement with the intrinsic X-ray flux emitted from the AGN, we have adopted an estimated intrinsic luminosity in the range of $\mathrm{L}_{1-100 \mathrm{keV}}$ $\sim 10^{43}-10^{43.5} \mathrm{erg} \mathrm{s}^{-1}$ (see, e.g. Colbert et al. 2002). Using the diameter of the CND of $4^{\prime \prime}$ and that of the SF-ring of $40^{\prime \prime}$, we derived an X-ray flux in the range of $\sim 3-10$ erg $\mathrm{s}^{-1} \mathrm{~cm}^{-2}$ and of $\sim 0.03-0.11$ erg $\mathrm{s}^{-1} \mathrm{~cm}^{-2}$, respectively for the compact and extended components.

The X-ray flux predicted for the XDR model of the CND component is in the range $1.6<\mathrm{F}_{X}<10 \mathrm{erg} \mathrm{s}^{-1} \mathrm{~cm}^{-2}$ (see right panel of Figure 7), therefore the hypothesis of an XDR origin of the intermediate-J CO lines in the CND can be considered valid. The X-ray flux predicted for the XDR model of the SF-ring of $\mathrm{F}_{X}=0.5-2.0 \mathrm{erg} \mathrm{s}^{-1} \mathrm{~cm}^{-2}$ is not in agreement with our estimates, being at least one order of magnitude higher, and therefore the XDR origin of the lower-J CO lines in the extended region can be excluded, given the assumption in the above paragraph. Moreover, even if the X-ray flux would be available to excite the XDR, e.g. in the case of an underestimated intrinsic X-ray luminosity, an unknown part of it is expected to be absorbed from the interstellar medium along the path, before reaching the SF-ring. The attenuation to the X-ray source as seen by the CO-emitting gas cannot in fact be quantified. Therefore the estimated flux could be considered as an upper limit.

Similarly we estimated the far-UV $(6-13.6 \mathrm{eV})$ flux from the AGN that illuminates the CND and the SF-ring. The adopted intrinsic far-UV luminosity of the AGN is $\sim 10^{42} \mathrm{erg} \mathrm{s}^{-1}$. This value was calculated using the in- trinsic far-UV continuum derived by Pier et al. (1994). At the distance of the SF-ring the far-UV Hux is $F_{\mathrm{FUV}}=$ $10^{0.5} \mathrm{G}_{0}$, whereas for the CND the $F_{\mathrm{FUV}}=10^{2.4} \mathrm{G}_{0}$.

This $F_{\mathrm{FUV}}$ for the CND implies a high-density $\left(n_{\mathrm{H}}>\right.$ $10^{5.5} \mathrm{~cm}^{-3}$ ) component according to the PDR models (left panel of Figure 7), which is not in agreement with the density estimate from the RADEX modeling. For the extended component, the UV flux from the AGN is lower than that predicted by the PDR models; however, in the star-forming ring it is likely that the UV emission from young stars also contributes to the interstellar UV radiation field. For comparison, the far-UV flux from a young stellar cluster (age $5 \mathrm{Myr}$, see Spinoglio et al. 2005) of stellar mass $10^{4} \mathrm{M}_{\odot}$ is $10^{2} \mathrm{G}_{0}$ at $50 \mathrm{pc}$. This far-UV flux is an order of magnitude higher than that from the AGN and would be compatible with the predictions of the PDR models (see left panel of Figure 11).

Taking into account all the above considerations, we therefore conclude that the most plausible explanation for the excitation of the CND is an XDR illuminated from the AGN, while that of the SF-ring is a PDR, mainly excited from the young stellar populations in the galactic arms.

\section{2. $\mathrm{H}_{2} \mathrm{O}$ emission}

We detected $6 \mathrm{H}_{2} \mathrm{O}$ transitions, 2 o- $\mathrm{H}_{2} \mathrm{O}$ and 4 p- $\mathrm{H}_{2} \mathrm{O}$ lines, above a $3 \sigma$ level within the SPIRE-FTS spectrum. In Table 1 we list the detected lines, reporting their frequency, upper level energy $E_{\text {up }} / \kappa_{B}$, and the measured flux. We also listed the water lines detected with PACS (S. Hailey-Dunsheath 2012, private communication). The line fluxes detected by PACS were measured with a Gaussian fitting of the line profile of the central spatial pixel (spaxel) and scaling using the standard point-source correction factors. The emission of all $\mathrm{H}_{2} \mathrm{O}$ lines detected in the SSW band of the SPIRE-FTS, as well as most of the PACS lines, are unresolved (diameter $\leq 20^{\prime \prime}$, for the SPIRE lines, see Figure 1, and $\leq 10^{\prime \prime}$, for the PACS lines), indicating that water emission arises mainly from the CND region. Only one line, the p$\mathrm{H}_{2} \mathrm{O} 2_{11}-2_{02}$ at $752.033 \mathrm{GHz}$ was observed in the SLW band, with a beam size of $\sim 33.4^{\prime \prime}$, and thus the emission of this transition may have an additional contribution from the starburst ring. However, our maps are not sensitive enough to disentangle the contribution of the starburst ring from the CND. Moreover, the PACS map of the $\mathrm{o}-\mathrm{H}_{2} \mathrm{O} 2_{12}-1_{01}$ line at $179 \mu \mathrm{m}(1669.905 \mathrm{GHz})$ shows extended emission (S. Hailey-Dunsheath private communication), most likely associated with the starburst ring. Thus, if there is some contribution from the extended emission in the central spaxel, the measured flux may overestimate the contribution of the nuclear flux.

\subsubsection{Excitation analysis}

In order to constrain the physical conditions of the water excitation, we built a grid of models using the Large Velocity Gradient (LVG) model in plane parallel geometry described in Ceccarelli et al. (1998) varying the parameters in the following range: $n\left(\mathrm{H}_{2}\right)=10^{3}-10^{8} \mathrm{~cm}^{-3}$, $T_{\text {kin }}=10-1500 \mathrm{~K}, N\left(\mathrm{o}-\mathrm{H}_{2} \mathrm{O}\right)$ and $N\left(\mathrm{p}-\mathrm{H}_{2} \mathrm{O}\right)$ from $10^{11}$ to $10^{17} \mathrm{~cm}^{-2}$. The molecular data were taken from the BASECOL ${ }^{15}$ database (Dubernet et al. 2006) and we

\footnotetext{
15 http://basecol.obspm.fr
} 

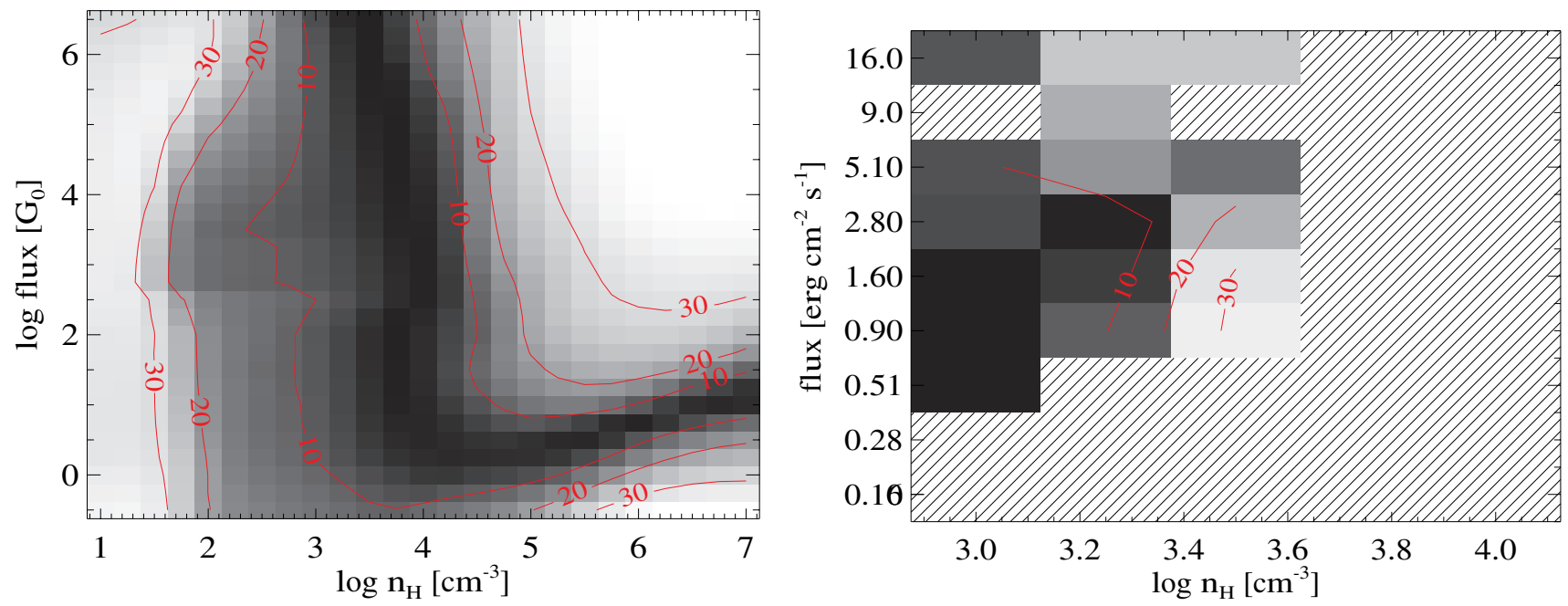

Fig. 11. - Reduced $\chi^{2}$ values of the PDR (Wolfire et al. 2010) and XDR models (Meijerink \& Spaans 2005 Meijerink et al. 2007) for the extended component. Left: PDR models; Right: XDR models. The line shaded region of the figure is where no model is available or the model does not predict the lines of interest.

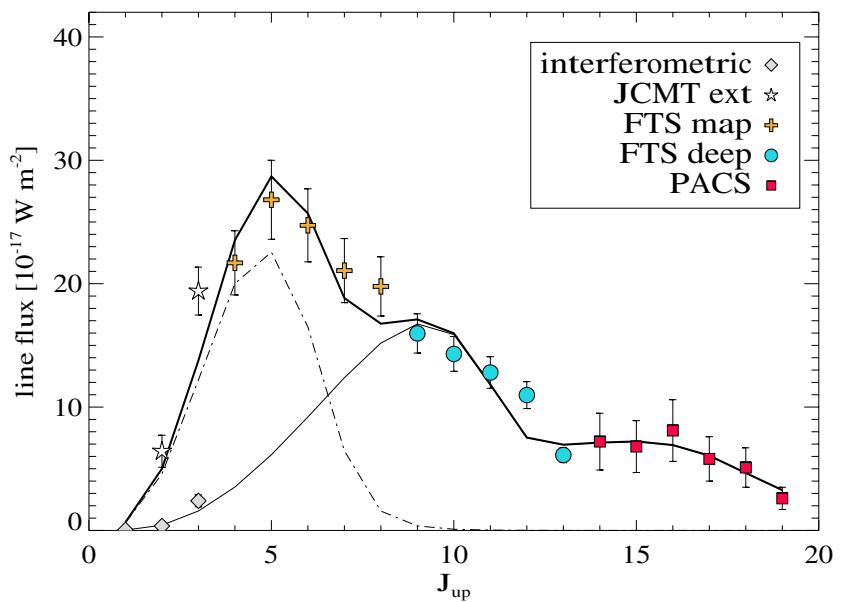

FIG. 12.- PDR/XDR model fitting for the extended component (SF-ring), together with the compact component, associated with the CND. Models have been taken from Meijerink \& Spaans (2005); Meijerink et al. (2007). Notations as in Figure 6 The crosses (yellow) represent the F"TS measurements from the spectral map and the stars (white) show the ground-based observations of the extended component (Kamenetzky et al. 2011, Wilson 2012, private communication). The thin solid lines show the fit of the compact component, which is the sum of the high- and mid-J models as in Figure 10 The dash-dotted lines show the fit of the extended component, with a PDR model with density $n\left(H_{2}\right)=10^{3.25} \mathrm{~cm}^{-3}$ and $\log G o=2.75$, once the compact component has been subtracted. The thick solid line shows the total modeled emission, which is compared to the observed data (see the text).

used the most recent collisional rate coefficients with $\mathrm{H}_{2}$ (Dubernet et al. 2009, Daniel et al. 2010, 2011), calculated for temperatures between $5 \mathrm{~K}$ and $1500 \mathrm{~K}$. The $\mathrm{H}_{2} \mathrm{O}$ spectrum has been computed considering 45 levels for the ortho and para water (i.e., considering the levels up to excitation temperatures of $\sim 2000 \mathrm{~K}$ ). The two water forms are treated as independent species. We used an ortho-to-para $\mathrm{H}_{2}$ ratio equal to 1 and an ortho-to-para $\mathrm{H}_{2} \mathrm{O}$ ratio of 1 . The model includes the effects of the beam filling factor, so it computes the $\chi^{2}$ for each column density minimizing with respect to the source size, temperature, and density. Our model does not include the effects of radiative pumping.

We fitted the $\mathrm{o}-\mathrm{H}_{2} \mathrm{O}$ and $\mathrm{p}-\mathrm{H}_{2} \mathrm{O}$ lines separately. We

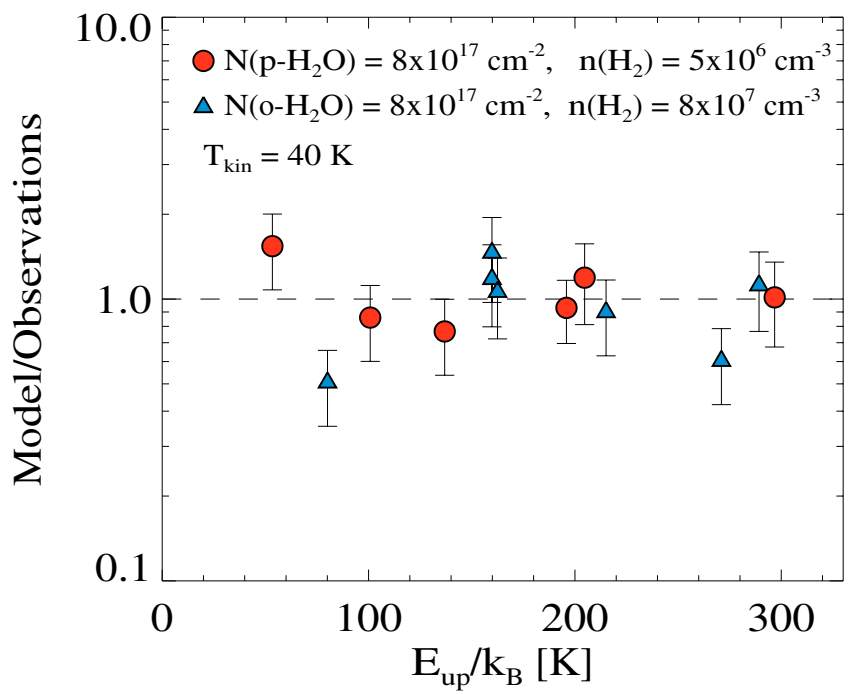

FiG. 13.- Ratio between the best fit model predictions and the observations as a function of the upper level energy. Triangles show the $\mathrm{o}-\mathrm{H}_{2} \mathrm{O}$ transitions and circles show the $\mathrm{p}-\mathrm{H}_{2} \mathrm{O}$ transitions. The best fit models predict a column density of both ortho- and para$\mathrm{H}_{2} \mathrm{O}$ of $\sim 8 \times 10^{17} \mathrm{~cm}^{-2}$ and hydrogen desities in the range $\mathrm{n}\left(\mathrm{H}_{2}\right)$ $=5 \times 10^{6}-8 \times 10^{7} \mathrm{~cm}^{-3}$.

found that the observations of water lines are consistent with LVG model predictions for an emission size of $10^{\prime \prime} \pm 2^{\prime \prime}$, a kinetic temperature of $T_{\text {kin }} \simeq 40 \pm 10 \mathrm{~K}$, a density $\mathrm{n}\left(\mathrm{H}_{2}\right)$ from $4 \times 10^{6}$ to $8 \times 10^{7} \mathrm{~cm}^{-3}$, and a value of the $N / \Delta v$ varying between $5 \times 10^{14}$ and $10^{16} \mathrm{~cm}^{-2}$. The column density of each water form can then be obtained once we know the linewidth, which we set to $\sim 100 \mathrm{~km} \mathrm{~s}^{-1}$. This translates into a column density for both o- $\mathrm{H}_{2} \mathrm{O}$ and $\mathrm{p}-\mathrm{H}_{2} \mathrm{O}$ around $5 \times 10^{16}-10^{18} \mathrm{~cm}^{-2}$. Figure 13 shows the best fit model for the $\mathrm{o}-\mathrm{H}_{2} \mathrm{O}$ and $\mathrm{p}-\mathrm{H}_{2} \mathrm{O}$ lines. The results of the LVG model predictions are summarized in Table4. As can be seen in Fig. 13, the best fit model predictions can reproduce most of the line fluxes reasonably well. Only for two o- $\mathrm{H}_{2} \mathrm{O}$ lines the LVG model underestimates the observed line flux. However, the line flux of the $\mathrm{o}-\mathrm{H}_{2} \mathrm{O} 22_{12}-1_{01}$ transition may be in fact overestimated due to some extended emission that may come 
from the starburst ring instead from the CND region (S. Hailey-Dunsheath private communication). As shown in previous sections and in Hailey-Dunsheath et al. (2012), the $\mathrm{CO}$ emission from the CND is associated with several components at different temperatures and densities. In the case of water we cannot rule out the presence of several components, but, due to the relatively low number of water lines, we did not attempt to fit the water lines with more than one component.

Our results suggest a posteriori that the excitation of the water lines is not strongly affected by radiative pumping. Without the inclusion of radiative pumping, we can indeed fit all the detected lines within a factor of two. Moreover the water transitions in the SPIRE FTS spectrum that should be more affected by radiative pumping, besides that of $\mathrm{o}-\mathrm{H}_{2} \mathrm{O} 3_{21}-3_{12}$, are those of $\mathrm{O}-\mathrm{H}_{2} \mathrm{O}$ $5_{23}-5_{14}$ and $\mathrm{p}-\mathrm{H}_{2} \mathrm{O} 4_{22}-4_{13}$ (see, e.g., González-Alfonso et al. 2010), which we do not detect, consistent with the LVG model predictions. On the other hand, we compare our findings with the results of Mrk 231 (GonzálezAlfonso et al. 2010), where all these three transitions have been detected and their fluxes can be reproduced only if radiative pumping is included. If we adopt the same line ratios of Mrk 231 for NGC 1068, we would expect to detect also these lines, originating from a higher level, at about a $10 \sigma$ level (assuming the $3 \sigma$ sensitivity in the SSW FTS range of $10^{-17} \mathrm{~W} \mathrm{~m}^{-2}$ ). We conclude therefore that for NGC 1068 collisions seem to be the dominant excitation mechanism for water and that radiative pumping can be neglected.

\subsection{Molecular ions of $\mathrm{H}_{2} \mathrm{O}^{+}, \mathrm{OH}^{+}$and $\mathrm{CH}^{+}$}

The molecular ions of $\mathrm{H}_{2} \mathrm{O}^{+}, \mathrm{OH}^{+}$, and $\mathrm{CH}^{+}$have been detected in emission in NGC 1068. The $1115 \mathrm{GHz}$ ground-state transition of $\mathrm{H}_{2} \mathrm{O}^{+}$, as well as the $1033 \mathrm{GHz}$ transition of $\mathrm{OH}^{+}$and that of $\mathrm{CH}^{+}$, have also been detected in emission in Mrk 231 (van der Werf et al. 2010). In contrast, these transitions have all been detected in absorption in Arp 220 (Rangwala et al. 2011) and two of them $\left(\mathrm{H}_{2} \mathrm{O}^{+}\right.$at $1115 \mathrm{GHz}$ and $\mathrm{OH}^{+}$at $\left.1033 \mathrm{GHz}\right)$ in absorption in M 82 (Kamenetzky et al. 2012).

To obtain the column densities of $\mathrm{H}_{2} \mathrm{O}^{+}, \mathrm{OH}^{+}$, and $\mathrm{CH}^{+}$, we assumed that the gas is in local thermodynamical equilibrium (LTE) and the emission is optically thin and originates in the CND region (diameter of $4^{\prime \prime}$ ). Assuming that all levels are populated according to the same excitation temperature, $T_{\text {ex }}$, the column density is given by (see, e.g., Goldsmith \& Langer 1999):

$$
N_{\mathrm{mol}}=\frac{4 \pi F Q\left(T_{\mathrm{ex}}\right) \exp \left(E_{\mathrm{up}} / k T_{\mathrm{ex}}\right)}{h \nu A_{\mathrm{ul}} g_{\mathrm{u}}}
$$

where $F$ is the integrated line brightness (in units of $\left.\operatorname{erg} \mathrm{cm}^{-2} \mathrm{~s}^{-1} \mathrm{sr}^{-1}\right), \nu$ is the frequency of the transition, $E_{\text {up }}$ the energy of the upper level, $Q\left(T_{\text {ex }}\right)$ is the partition function at the excitation temperature $T_{\mathrm{ex}}$, and $h$ and $k$ are the Planck and Boltzmann constants, respectively. We approximated the partition function of $\mathrm{CH}^{+}$ and $\mathrm{OH}^{+}$to $Q_{\text {rot }} \simeq k T_{\mathrm{ex}} / h B$, where $B$ is the rotational constant of the molecule. Einstein coefficients $A_{\mathrm{ul}}$, upper level degeneracy $g_{\mathrm{u}}$, and rotational constants have been obtained from the Cologne Database for Molecular Spectroscopy (CDMS; Müller et al. 2001, 2005). For $\mathrm{O}-\mathrm{H}_{2} \mathrm{O}^{+}$the partition function has been obtained using the energy levels listed in the database. Both $\mathrm{H}_{2} \mathrm{O}^{+}$and $\mathrm{OH}^{+}$present hyperfine structure, which is not resolved in the SPIRE FTS spectrum. Therefore, we used $\sum_{i} A_{i 1} g_{i}$ to account for the several hyperfine components. We adopted an ortho-to-para ratio for $\mathrm{H}_{2} \mathrm{O}^{+}$of 3 .

The estimated column densities are $N\left(\mathrm{H}_{2} \mathrm{O}^{+}\right)=(2.0-$ $5.9) \times 10^{13} \mathrm{~cm}^{-2}, N\left(\mathrm{OH}^{+}\right)=(1.0-1.2) \times 10^{13} \mathrm{~cm}^{-2}$, and $N\left(\mathrm{CH}^{+}\right)=(5.4-7.5) \times 10^{13} \mathrm{~cm}^{-2}$, for a range of excitation temperatures between 40 and $100 \mathrm{~K}$. The uncertainty in the derived column densities is estimated to be around $50 \%$, which arises from the uncertainty in the flux calibration. The $\mathrm{p}-\mathrm{H}_{3} \mathrm{O}^{+} J_{\mathrm{K}}=3_{2}^{+}-2_{2}^{-}$line at $364.7974 \mathrm{GHz}$ has been detected in NGC 1068 using the JCMT (Aalto et al. 2011). We recomputed the $\mathrm{H}_{3} \mathrm{O}^{+}$column density using Eq. 1. our adopted size of the CND region of $4^{\prime \prime}$, and the excitation temperature range of $T_{\mathrm{ex}} \sim 40-100 \mathrm{~K}$. We obtained $N\left(\mathrm{p}-\mathrm{H}_{3} \mathrm{O}^{+}\right) \simeq(1.8-0.7) \times 10^{14} \mathrm{~cm}^{-2}$. Assuming an intermediate ortho-to-para ratio of 1.5 van der Tak et al. 2008), the total column density of $\mathrm{H}_{3} \mathrm{O}^{+}$ is $\sim(4.5-1.8) \times 10^{14} \mathrm{~cm}^{-2}$.

Considering the spectrum of Arp 220 presented in Rangwala et al. (2011), we notice that in NGC 1068, the ratio of $\mathrm{N}\left(\mathrm{H}_{3} \mathrm{O}^{+}\right) / \mathrm{N}\left(\mathrm{H}_{2} \mathrm{O}^{+}\right) \sim 10$, whereas this ratio is reversed in Arp 220. Similarly, the inferred column densities indicate that $\mathrm{N}\left(\mathrm{H}_{3} \mathrm{O}^{+}\right) / \mathrm{N}\left(\mathrm{OH}^{+}\right) \gg 1$ in $\mathrm{NGC} 1068$, while in Arp 220 the $\mathrm{OH}^{+}$column density is at least an order of magnitude larger than that of $\mathrm{H}_{3} \mathrm{O}^{+}$. So the relative abundances of the molecular ions appear to be very different in the two galaxies, suggesting some substantially different conditions.

We compared our estimated column densities with the predictions of the Hollenbach et al. (2012) PDR models for different FUV incident fluxes and cosmic ray ionization rates. For the FUV radiation field produced by the $\mathrm{AGN}$ in the CND region (see Section 3.1.4) the order of magnitude of the observed column densities of $\mathrm{H}_{2} \mathrm{O}^{+}$ and $\mathrm{OH}^{+}$are compatible with a low density medium (see Fig. 9 and Fig. 10 of Hollenbach et al. (2012)). According to their models, the column density of $\mathrm{H}_{3} \mathrm{O}^{+}$is approximately constant with a value of $10^{14} \mathrm{~cm}^{-2}$, in agreement with our estimated value. Combining the observed value of the $N\left(\mathrm{OH}^{+}\right) / N\left(\mathrm{H}_{2} \mathrm{O}^{+}\right)$ratio and the value of $N\left(\mathrm{OH}^{+}\right)$, we estimated a range of values of $\log _{10}[\zeta / n]$ between -15.5 and -16.5 , where $\zeta$ is the cosmic ray ionization rate and $n$ is the hydrogen nucleus density, and we derived $N_{\mathrm{H}}>2 \times 10^{20} \mathrm{~cm}^{-2}$ (see Figs. 10 to 13 of Hollenbach et al. (2012)). Therefore PDR models which include cosmic rays are in agreement with the observed column densities of these molecular ions.

\subsection{Hydrogen fluoride}

Herschel spectroscopic observations have detected for the first time the $\mathrm{J}=1-0$ transition of hydrogen fluoride (HF) at $1232 \mathrm{GHz}$ in the local universe, and revealed the ubiquitous nature of this molecule in the interstellar medium (ISM) of the Milky Way and of a few extragalactic sources, such as the local AGN/ULIRGs Mrk 231 (van der Werf et al. 2010), Arp 220 (Rangwala et al. 2011), M82 (Kamenetzky et al. 2012) and in the Coverleaf quasar at $\mathrm{z}=2.56$ (Monje et al. 2011).

This transition is generally observed in absorption, as expected, due to its very large Einstein coefficient, $\mathrm{A}_{10}=$ $2.42 \times 10^{-2} \mathrm{~s}^{-1}$. Only an extremely dense region, with a 
strong radiation field, could generate enough excitation to yield an $\mathrm{HF}$ feature in emission (Neufeld \& Wolfire 2009). In NGC 1068 it has been detected in emission (at a S/N > 10), similarly to Mrk 231 and in contrast with Arp 220, where it has been detected in absorption.

We estimated the column density of hydrogen fluoride using Equation 1 assuming that the HF emission originated in the $\mathrm{CND}$ region (diameter $\sim 4^{\prime \prime}$ ). We adopted the kinetic temperature $T_{\text {kin }} \simeq 100 \mathrm{~K}$ derived for the $\mathrm{CO}$ gas associated with the CND region (see Sec.3.2.1) as an upper limit to the excitation temperature. The values of the upper level degeneracy, $g_{\text {up }}=3$, and the rotational constant $(B=616365 \mathrm{MHz})$ to calculate the partition function at a given temperature, $Q_{\text {rot }} \simeq k T_{\mathrm{ex}} / h B$, have been taken from the Jet Propulsion Laboratory (JPL) catalog (Pickett et al. 1998). For $T_{\text {ex }}=40-100 \mathrm{~K}$, we found a column density of $\mathrm{HF}$ of $N(\mathrm{HF}) \simeq 7 \times 10^{12} \mathrm{~cm}^{-2}$. This value is one order of magnitude lower than the $N(\mathrm{HF})$ value found in M82 (Kamenetzky et al. 2012), where hydrogen fluoride is seen in absorption. Calculations with RADEX indicate that the optically thin approximation holds for a wide range of physical conditions, with $\tau \ll 1$ for temperatures between $T_{\text {kin }}=20-100 \mathrm{~K}$, densities $n\left(\mathrm{H}_{2}\right) \simeq 10^{3}-10^{6} \mathrm{~cm}^{-3}$, and column densities of $10^{11}-10^{14} \mathrm{~cm}^{-2}$.

\subsection{Atomic lines}

In this section, we analyze the origin of the three atomic fine-structure lines detected with the SPIRE FTS, namely, the two lines from neutral carbon and the [NII] $205 \mu \mathrm{m}$ line. First we attempt to use the composite photoionization model used by Spinoglio et al. (2005), which successfully fitted the overall UV to far-IR spectrum of NGC 1068 reproducing the line fluxes within a factor 2 on average. This model was composed of an AGN component and a starburst component, which included contribution from PDR clouds, as the integration was allowed to run until the gas temperature in the cloud cooled down to $\mathrm{T}=50 \mathrm{~K}$. We compare in the next section the predictions of the atomic lines detected by the SPIRE FTS using the same photoionization model reported in Spinoglio et al. (2005), which were not reported in that work.

\subsection{1. [NII] and [CI] emisson}

The observed [NII] $205 \mu \mathrm{m}$ line is roughly consistent with the photoionization model presented in Spinoglio et al. (2005). The predicted ratio [NII] $205 \mu \mathrm{m} / 122 \mu \mathrm{m}$ is $\sim 0.47$, while the observed value is $18.8 / 30.5=0.62 \pm 0.09$, using the ISO-LWS observation of the [NII]122 $\mu \mathrm{m}$ line (Spinoglio et al. 2005). Taking into account that the two lines have been observed by two different instruments, with different apertures, this result is satisfactory. We can therefore conclude that the diffuse ionized emission traced by the [NII] ion is in agreement with being excited by the starburst component in the SF-ring of NGC 1068. The observed [CI]369 $\mu \mathrm{m} /[\mathrm{CI}] 609 \mu \mathrm{m}$ line ratio is $1.65 \pm 0.30$, while the ratio predicted by the photoionization model is about 3.8, indicating that the emission of the line at $609 \mu \mathrm{m}$ could be more extended than the FTS beam.

On the other hand, the intensity of the [CI] lines predicted by the photoionization model are weaker by more than an order of magnitude compared to the observed values relatively to the $[\mathrm{CII}] 158 \mu \mathrm{m}$ emission, as measured in Spinoglio et al. (2005). The predicted ratio of $[\mathrm{CI}] 369 \mu \mathrm{m} /[\mathrm{CII}] 158 \mu \mathrm{m}$ is in fact $\sim 10^{-3}$ and the predicted [CI] $609 /[\mathrm{CII}] 158 \mu \mathrm{m} \mu \mathrm{m}$ is $\sim 3 \times 10^{-4}$, while the observed ratios are $9.7 \times 10^{-3}$ and $5.9 \times 10^{-3}$, respectively. This discrepancy between data and models could be due to the poor ability of current models to reproduce the [CI] emission line intensities (see, e.g., Röllig et al. 2007).

\subsubsection{Using Neutral Carbon emission to estimate the gas temperature}

We derive here the kinetic temperature of the molecular gas from the ratio of the intensities of the neutral Carbon lines ([CI] at 369 and $609 \mu \mathrm{m})$, when local thermodynamic equilibrium (LTE) is assumed. The Boltzmann equation can be written as

$$
T_{k}=\frac{-E_{21}}{k}\left[\ln \left(\frac{g_{1} n_{2}}{g_{2} n_{1}}\right)\right]^{-1}
$$

where $T_{k}$ is the kinetic temperature of the gas, $E_{21}=$ $E_{2}-E_{1}, n_{1}$ and $n_{2}$ are the level populations and $g_{1}$ and $g_{2}$ their statistical weights. The integrated flux in a line is simply $F_{i j} \propto A_{i j} n_{i} \nu_{i j}$. Therefore the kinetic temperature can be written as

$$
T_{k}=\frac{-E_{21}}{k}\left[\ln \left(\frac{g_{1}}{g_{2}} \cdot \frac{A_{10} \nu_{10} F_{21}}{A_{21} \nu_{21} F_{10}}\right)\right]^{-1}
$$

By substituting the observed values of the fluxes of the [CI] lines at 492.16 and $609.34 \mathrm{GHz}$ of Table 1 and propagating the uncertainties, we obtain a kinetic temperature of the gas of $\mathrm{T}_{k}=22.5 \pm 2.4 \mathrm{~K}$. The kinetic temperature derived from the two [CI] lines is much lower than for either the CND or extended starburst ring components, which again argues that the CI emission is much more extended than either of these.

\subsection{Comparing the derived masses with previous work}

We discuss here our results in the context of the various molecular observations of NGC 1068 in the literature. In particular our radiation transfer models indicate the presence of two major components responsible for the $\mathrm{CO}$ $\left(\mathrm{J}_{u p} \leq 13\right)$ excitation: the first one is a compact component, with a diameter of $\sim 4^{\prime \prime}(300 \mathrm{pc})$, associated with the CND with a density of $\mathrm{n}\left(\mathrm{H}_{2}\right) \sim 4 \times 10^{4} \mathrm{~cm}^{-3}$, kinetic temperature of $\mathrm{T} \sim 90 \mathrm{~K}$ and mass $\mathrm{M}\left(\mathrm{H}_{2}\right) \sim 2.4 \times 10^{7} \mathrm{M}_{\odot}$, and the second is an extended component, with a diameter of $\sim 40^{\prime \prime}(3 \mathrm{kpc})$, associated with the SF-ring, with a density of $\mathrm{n}\left(\mathrm{H}_{2}\right) \sim 7 \times 10^{2} \mathrm{~cm}^{-3}$, kinetic temperature of $\mathrm{T} \sim 116 \mathrm{~K}$ and mass $\mathrm{M}\left(\mathrm{H}_{2}\right) \sim 3.5 \times 10^{8} \mathrm{M}_{\odot}$.

Schinnerer et al. (2000) have used interferometric CO (1-0) and (2-1) observations to derive a mass of the CND ("ring" in CO (2-1), in their terminology, of $\sim 200 \mathrm{pc}$ in radius) of $\mathrm{M}\left(\mathrm{H}_{2}\right) \sim 5 \times 10^{7} \mathrm{M}_{\odot}$, while they measure a mass of the "spiral arms" (our SF-ring) of $\mathrm{M}\left(\mathrm{H}_{2}\right) \sim$ $6.8 \times 10^{8} \mathrm{M}_{\odot}$. These two mass estimates are a factor 2 times larger than our estimates; however, this discrepancy is fully justified by the difference in the gas excitation, as the CO (1-0) and (2-1) lines are mapping lower temperature gas. 
We then compare our findings with the temperature of $\mathrm{T} \geq 145 \mathrm{~K}$ and mass derived from the $\mathrm{H}_{2}$ pure rotational transitions of Rigopoulou et al. (2002); from the detection of the $\mathrm{S}(1)$ line (Lutz et al. 2000) a warm gas mass of $\mathrm{M}\left(\mathrm{H}_{2}\right) \sim 10^{8} \mathrm{M}_{\odot}$ is derived in the SWS beam of $14^{\prime \prime}$ $\times 27^{\prime \prime}$ (de Graauw et al. 1996). This is consistent with our findings, given the difference in beam size between the two spectrometers.

\subsection{Observability of submillimetre lines at high redshift}

Using NGC 1068 as a template to predict the submillimetre spectrum of higher redshift galaxies, we have estimated the expected line fluxes as a function of redshift, rescaling the total infrared luminosity of NGC 1068 to the value of $\mathrm{L}_{I R}=10^{12} \mathrm{~L}_{\odot}$. We show in Figure $14(\mathrm{a})$ the observed fluxes of atomic and molecular lines of NGC 1068 from the ISO-LWS spectrometer (Spinoglio et al.|2005), from the PACS spectrometer (Hailey-Dunsheath et al. 2012), and those from this work, and compare them with the predicted fluxes at redshift of $\mathrm{z}=0.1,0.2,0.3,0.5$ and $1,2,3,5$. We also show in the figure the $5 \sigma 1$ hour sensitivities of future observing facilities, such as CCAT and SPICA-SAFARI and compare them with the sensitivities of the Herschel spectrometers. We refer to Spinoglio et al. (2012) and references therein for a brief description of SPICA and CCAT and for the details of the expected sensitivities of the foreseen spectrometers at their focal planes. We also show in Figure 14(b), for comparison with NGC 1068, the observed and predicted submillimetre spectra of other important local template galaxies, the prototypical starburst galaxy M82 and the ULIRG Arp220, that have been observed with the SPIRE spectrometer (Kamenetzky et al. 2012, Rangwala et al. 2011, respectively).

It is clear from Figure 14 that the intermediate to highJ CO lines, as well as the two [CI] lines, are weaker by 1-2 orders of magnitude compared to the brightest far-IR fine structure lines of [CII] and [NII]. However, their powerful diagnostic potential, in terms of detecting through XDR regions the effect of an AGN in the host galaxy, combined with their long wavelengths, makes these lines very attractive for high redshift spectroscopic cosmological surveys from ground-based telescopes. The expected sensitivity of future spectrometers at the focal plane of large submillimetre telescopes, such as CCAT, will be able to detect an object like NGC 1068, M82, or Arp220 with a luminosity of $\mathrm{L}=10^{12} \mathrm{~L}_{\odot}$ at a redshift of $\mathrm{z}=0.2-0.5$.

\section{SUMMARY AND CONCLUSIONS}

We summarize here the results of this work. The first complete submillimetre $(190-670 \mu \mathrm{m})$ spectrum of the Seyfert type 2 galaxy NGC 1068 reveals the full sequence of $\mathrm{CO}$ pure rotational lines from $\mathrm{J}_{u p}=4$ to $\mathrm{J}_{u p}=13$. The radiation transfer analysis of these lines shows the presence of two physically distinct components: the first one originating from the circum-nuclear disk (CND) of few arcseconds in diameter $\left(\sim 4^{\prime \prime}\right)$ and the second one excited in the star forming ring (SF-ring) with a diameter ten times larger $\left(\sim 40^{\prime \prime}\right)$. These results indicate a kinetic temperature of $\mathrm{CO}$ of $T_{\text {kin }}=100 \mathrm{~K}$ and $127 \mathrm{~K}$, a gas density of $n\left(\mathrm{H}_{2}\right)=10^{4.5}$ and $10^{2.9} \mathrm{~cm}^{-3}$ and a derived molecular hydrogen mass of $\mathrm{M}\left(\mathrm{H}_{2}\right) \sim 2.4 \times 10^{7} \mathrm{M}_{\odot}$ and $\mathrm{M}\left(\mathrm{H}_{2}\right) \sim 3.5 \times 10^{8} \mathrm{M}_{\odot}$, for the compact and extended regions, respectively.
The comparison of the observed CO line intensities with predictions of photodissociation (PDR) and X-ray dominated regions (XDR) models shows that the circumnuclear disk emission can be modeled equally well by both types of models, while the CO lines in the starforming ring can be modeled by a photodissociation region only. However some observational constraints, such as the total CO surface brightness and the required radiation field, indicate that the most plausible explanation for the CO excitation of the CND is an XDR with density of $\mathrm{n}\left(\mathrm{H}_{2}\right) \sim 10^{4} \mathrm{~cm}^{-3}$ and X-ray flux of $9 \mathrm{erg} \mathrm{s}^{-1} \mathrm{~cm}^{-2}$, consistent with the AGN illumination. In contrast, the excitation of the SF-ring component is due to PDR emission originating from the young stars/HII regions in the spiral arms.

The water lines that we have detected with SPIRE, together with those observed by PACS (S. HaileyDunsheath 2012, private comm.), have been modeled with an LVG model to constrain the physical conditions of the water excitation. We have found that the kinetic temperature is $\mathrm{T}_{k i n}=40 \mathrm{~K}$, the molecular hydrogen density is $n\left(H_{2}\right)=4 \times 10^{6}-8 \times 10^{7}$ and the column density is of order $\mathrm{N}\left(\mathrm{H}_{2} \mathrm{O}\right)=8 \times 10^{17} \mathrm{~cm}^{-2}$ for both water forms.

The computed column densities of the molecular ions detected $\left(\mathrm{H}_{2} \mathrm{O}^{+}\right.$and $\left.\mathrm{OH}^{+}\right)$are in agreement with PDR models that include cosmic ray ionization.

The fundamental rotational transition of $\mathrm{HF}$ has been detected in emission in NGC 1068 and we infer a column density of $\mathrm{N}(\mathrm{HF}) \sim 7.5 \times 10^{12} \mathrm{~cm}^{-2}$.

For the two [CI] transitions, we derived a kinetic temperature of $22.5 \pm 2.4 \mathrm{~K}$ in LTE approximation, which is much lower than the temperatures traced from the intermediate-J CO molecular gas.

The molecular masses that we derived from our analysis are in good agreement with the masses estimated from both CO interferometric measurements of low-J lines and mid-infrared $\mathrm{H}_{2}$ emission lines.

Finally we show that the intermediate-J CO and [CI] lines in galaxies with $\mathrm{L} \sim 10^{12} L_{\odot}$ can be observed from planned and future ground-based and space telescopes up to redshift of $\mathrm{z} \sim 0.5$, making their diagnostic power an important tool to study galaxy evolution at intermediate redshift.

We thank Nicola Sacchi for having provided a preliminary reduction and analysis of the SPIRE FTS spectrum of NGC 1068, Steven Hailey-Dunsheath for having provided to us PACS data before publication and Scige Liu for assistance in the SPIRE data reduction. We also thank the anonymous referee for his/her careful analysis of the paper that helped improving it.

SPIRE has been developed by a consortium of institutes led by Cardiff Univ. (UK) and including: Univ. Lethbridge (Canada); NAOC (China); CEA, LAM (France); IFSI, Univ. Padua (Italy); IAC (Spain); Stockholm Observatory (Sweden); Imperial College London, RAL, UCL-MSSL, UKATC, Univ. Sussex (UK); and Caltech, JPL, NHSC, Univ. Colorado (USA). This development has been supported by national funding agencies: CSA (Canada); NAOC (China); CEA, CNES, CNRS (France); ASI (Italy); MCINN (Spain); SNSB (Sweden); STFC, UKSA (UK); and NASA (USA). This work has been funded in Italy from ASI (Italian Space Agency) 

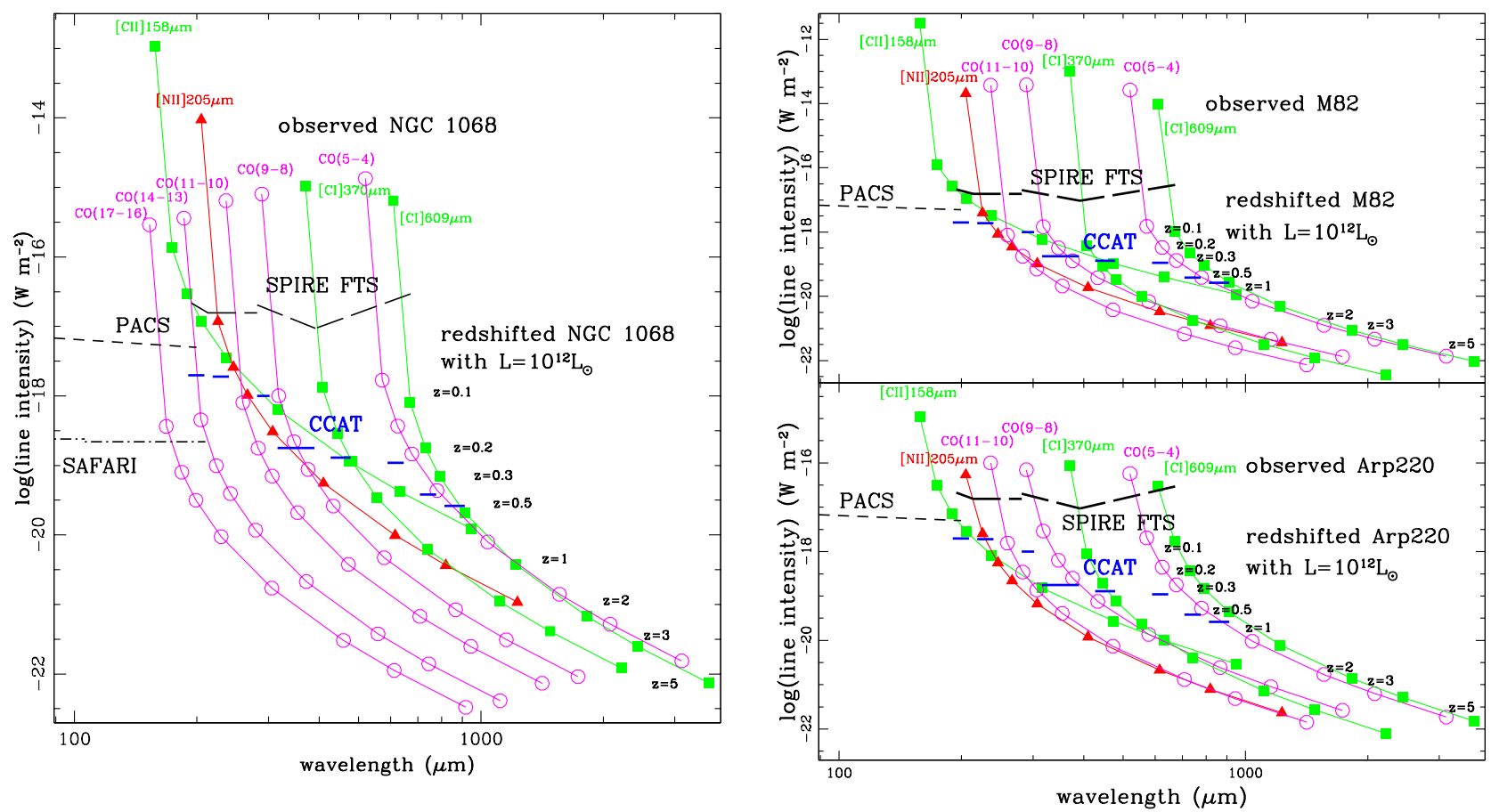

FIG. 14. - Left panel: Comparison between the observed far-IR/submillimetre lines of NGC 1068 with the predictions of the same lines as a function of redshift of a similar galaxy with an intrinsic luminosity of $\mathrm{L}=10^{12} \mathrm{~L} \odot$. The stellar/HII region lines are shown as filled triangles (in red), the low-ionization/PDR lines are shown as filled squares (in green), while the molecular lines are shown as open circles (in magenta). For comparison, we overplot the $5 \sigma$ (in $1 \mathrm{hr}$ ) sensitivity threshold of Herschel-PACS, Herschel-SPIRE, SPICA-SAFARI and CCAT. See Spinoglio et al. (2012) for more details on the assumed sensitivities of the future SAFARI and CCAT instruments. Right panel: Same diagram for other two templates: the starburst galaxy M82 (Kamenetzky et al.|2012) and the ULIRG Arp220 (Rangwala et al.|2011).

under contract I/005/11/0. The research of C.D.W. is supported by grants from NSERC (Canada).

\section{REFERENCES}

Aalto, S., Costagliola, F., van der Tak, F., \& Meijerink, R. 2011, A\&A, 527, A69

Antonucci, R. 1993, ARA\&A, 31, 473

Antonucci, R. R. J., \& Miller, J. S. 1985, ApJ, 297, 621

Bendo, G. J., et al. 2012, MNRAS, 419, 1833

Bland-Hawthorn, J., Gallimore, J. F., Tacconi, L. J., Brinks, E., Baum, S. A., Antonucci, R. R. J., \& Cecil, G. N. 1997, Ap\&SS, 248,9

Ceccarelli, C., et al. 1998, A\&A, 331, 372

Clegg, P. E., et al. 1996, A\&A, 315, L38

Colbert, E. J. M., Weaver, K. A., Krolik, J. H., Mulchaey, J. S., \& Mushotzky, R. F. 2002, ApJ, 581, 182

Daniel, F., Dubernet, M.-L., \& Grosjean, A. 2011, A\&A, 536, A76

Daniel, F., Dubernet, M.-L., Pacaud, F., \& Grosjean, A. 2010, A\&A, 517, A13

de Graauw, T., et al. 1996, A\&A, 315, L49

Draine, B. T. 1978, ApJS, 36, 595

Dubernet, M.-L., Daniel, F., Grosjean, A., \& Lin, C. Y. 2009, A\&A, 497, 911

Dubernet, M.-L., Grosjean, A., Flower, D., Roueff, E., Daniel, F., Moreau, N., \& Debray, B. 2006, Journal of Plasma Research SERIES, Volume 7, p. 356-357, 7, 356

Fulton, T. R., et al. 2010, in Society of Photo-Optical Instrumentation Engineers (SPIE) Conference Series, Vol. 7731, Society of Photo-Optical Instrumentation Engineers (SPIE) Conference Series

Gallimore, J. F., Baum, S. A., \& O'Dea, C. P. 2004, ApJ, 613, 794

García-Burillo, S., et al. 2010, A\&A, 519, A2

Goldsmith, P. F. and Langer, W. D.1999, ApJ, 517, 209

González-Alfonso, E., et al. 2010, A\&A, 518, L43

Griffin, M. J., et al. 2010, A\&A, 518, L3

Hailey-Dunsheath, S., et al. 2012, ArXiv e-prints

Hollenbach, D., Kaufman, M. J., Neufeld, D., Wolfire, M., \&

Goicoechea, J. R. 2012, eprint arXiv:1205.6446

Jaffe, W., et al. 2004, Nature, 429, 47

Kamenetzky, J., et al. 2011, ApJ, 731, 83
—. 2012, ApJ, 753, 70

Kaufman, M. J., Wolfire, M. G., \& Hollenbach, D. J. 2006, ApJ, 644,283

Kessler, M. F., et al. 1996, A\&A, 315, L27

Kohno, K., Nakanishi, K., Tosaki, T., Muraoka, K., Miura, R., Ezawa, H., \& Kawabe, R. 2008, Ap\&SS, 313, 279

Krips, M., Eckart, A., Neri, R., Schödel, R., Leon, S., Downes, D., García-Burillo, S., \& Combes, F. 2006, A\&A, 446, 113

Krips, M., et al. 2011, ApJ, 736, 37

Krips, M., Neri, R., García-Burillo, S., Martín, S., Combes, F., Graciá-Carpio, J., \& Eckart, A. 2008, ApJ, 677, 262

Lutz, D., Sturm, E., Genzel, R., Moorwood, A. F. M., Alexander, T., Netzer, H., \& Sternberg, A. 2000, ApJ, 536, 697

Maloney, P. R., Hollenbach, D. J., \& Tielens, A. G. G. M. 1996, ApJ, 466, 561

Meijerink, R., \& Spaans, M. 2005, A\&A, 436, 397

Meijerink, R., Spaans, M., \& Israel, F. P. 2007, A\&A, 461, 793

Monje, R. R., Phillips, T. G., Peng, R., Lis, D. C., Neufeld, D. A., \& Emprechtinger, M. 2011, ApJ, 742, L21

Müller, H. S. P., Schlöder, F., Stutzki, J., \& Winnewisser, G. 2005, Journal of Molecular Structure, 742, 215

Müller, H. S. P., Thorwirth, S., Roth, D. A., \& Winnewisser, G. 2001, A\&A, 370, L49

Müller Sánchez, F., Davies, R. I., Genzel, R., Tacconi, L. J., Eisenhauer, F., Hicks, E. K. S., Friedrich, S., \& Sternberg, A. 2009, ApJ, 691, 749

Naylor, B. J., et al. 2010a, ApJ, 722, 668

Naylor, D. A., et al. 2010b, in Society of Photo-Optical

Instrumentation Engineers (SPIE) Conference Series, Vol. 7731,

Society of Photo-Optical Instrumentation Engineers (SPIE) Conference Series

Neufeld, D. A., \& Wolfire, M. G. 2009, ApJ, 706, 1594

Pickett, H. M., Poynter, R. L., Cohen, E. A., Delitsky, M. L.,

Pearson, J. C., \& Müller, H. S. P. 1998,

J. Quant. Spec. Radiat. Transf., 60, 883 
Pier, E. A., Antonucci, R., Hurt, T., Kriss, G., \& Krolik, J. 1994, ApJ, 428, 124

Pilbratt, G. L., et al. 2010, A\&A, 518, L1

Poglitsch, A., et al. 2010, A\&A, 518, L2

Rangwala, N., et al. 2011, ApJ, 743, 94

Rigopoulou, D., Kunze, D., Lutz, D., Genzel, R., \& Moorwood, A. F. M. 2002, A\&A, 389, 374

Röllig, M., et al. 2007, A\&A, 467, 187

Rotaciuc, V., Krabbe, A., Cameron, M., Drapatz, S., Genzel, R., Sternberg, A., \& Storey, J. W. V. 1991, ApJ, 370, L23

Schinnerer, E., Eckart, A., Tacconi, L. J., Genzel, R., \& Downes, D. 2000, ApJ, 533, 850

Schöier, F. L., van der Tak, F. F. S., van Dishoeck, E. F., \& Black, J. H. 2005, A\&A, 432, 369

Scoville, N. Z., Matthews, K., Carico, D. P., \& Sanders, D. B. 1988, ApJ, 327, L61

Spinoglio, L., Dasyra, K. M., Franceschini, A., Gruppioni, C., Valiante, E., \& Isaak, K. 2012, ApJ, 745, 171

Spinoglio, L., Malkan, M. A., Smith, H. A., González-Alfonso, E., \& Fischer, J. 2005, ApJ, 623, 123
Tacconi, L. J., Genzel, R., Blietz, M., Cameron, M., Harris, A. I., \& Madden, S. 1994, ApJ, 426, L77

Thatte, N., Quirrenbach, A., Genzel, R., Maiolino, R., \& Tecza, M. 1997, ApJ, 490, 238

Thronson, Jr., H. A., et al. 1989, ApJ, 343, 158

Tsai, M., Hwang, C.-Y., Matsushita, S., Baker, A. J., \& Espada, D. 2012, ApJ, 746, 129

Usero, A., García-Burillo, S., Fuente, A., Martín-Pintado, J., \&

Rodríguez-Fernández, N. J. 2004, A\&A, 419, 897

van der Tak, F. F. S., Aalto, S., \& Meijerink, R. 2008, A\&A, 477, L5

van der Tak, F. F. S., Black, J. H., Schöier, F. L., Jansen, D. J., \& van Dishoeck, E. F. 2007, A\&A, 468, 627

van der Werf, P. P., et al. 2010, A\&A, 518, L42

Ward, J. S., Zmuidzinas, J., Harris, A. I., \& Isaak, K. G. 2003, ApJ, 587, 171

Wolfire, M. G., Hollenbach, D., \& McKee, C. F. 2010, ApJ, 716, 1191

Yang, B., Stancil, P. C., Balakrishnan, N., \& Forrey, R. C. 2010, ApJ, 718, 1062 
TABLE 1

Fluxes of the molecular and ATOMic Lines DeteCted in the SPIRE FTS SPECTRum of NGC 1068 AND COMPARED tO LITERATURE DATA

\begin{tabular}{|c|c|c|c|c|c|c|c|c|}
\hline \multirow[t]{2}{*}{ species } & \multirow[t]{2}{*}{ line id. } & \multirow{2}{*}{$\begin{array}{c}\text { rest. freq. } \\
(\mathrm{GHz})\end{array}$} & \multirow{2}{*}{$\begin{array}{c}E_{\mathrm{up}} / \kappa_{B} \\
(\mathrm{~K})\end{array}$} & \multirow{2}{*}{$\begin{array}{c}\text { Beam } \\
\text { FWHM } \\
\left({ }^{\prime \prime}\right)\end{array}$} & Flux & $1 \sigma$ Stat. Uncert. & $1 \sigma$ Tot. Uncert. & \multirow[t]{2}{*}{ 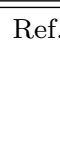 } \\
\hline & & & & & \multicolumn{3}{|c|}{$\left(10^{-17} \mathrm{~W} \mathrm{~m}^{-2}\right)$} & \\
\hline \multicolumn{9}{|c|}{ compact source } \\
\hline${ }^{12} \mathrm{CO}$ & $\begin{array}{l}\mathrm{J}=1-0 \\
\mathrm{~J}=2-1 \\
\mathrm{~J}=3-2 \\
\mathrm{~J}=9-8 \\
\mathrm{~J}=10-9 \\
\mathrm{~J}=11-10 \\
\mathrm{~J}=12-11 \\
\mathrm{~J}=13-12\end{array}$ & $\begin{array}{r}115.271 \\
230.538 \\
345.796 \\
1036.912 \\
1151.985 \\
1267.014 \\
1381.995 \\
1496.923\end{array}$ & $\begin{array}{c}5.53 \\
16.60 \\
33.19 \\
248.88 \\
304.16 \\
364.97 \\
431.29 \\
503.13\end{array}$ & $\begin{array}{c}1 \times 0.8 \\
1 \times 0.8 \\
1 \times 0.8 \\
18.7 \\
17.1 \\
17.6 \\
16.9 \\
16.8\end{array}$ & $\begin{array}{c}.050 \\
.360 \\
2.36 \\
15.41 \\
14.32^{\mathrm{a}} \\
12.80 \\
10.97 \\
6.11\end{array}$ & $\begin{array}{l}.001 \\
.003 \\
0.34 \\
0.15 \\
0.23 \\
0.15 \\
0.18 \\
0.27\end{array}$ & $\begin{array}{l}0.01 \\
0.07 \\
0.50 \\
1.55 \\
1.45 \\
1.29 \\
1.11 \\
0.67\end{array}$ & $\begin{array}{l}1 \\
1 \\
1 \\
2 \\
2 \\
2 \\
2 \\
2\end{array}$ \\
\hline $\mathrm{o}-\mathrm{H}_{2} \mathrm{O}$ & $\begin{array}{l}3_{12}-3_{03} \\
3_{21}-3_{12} \\
2_{21}-2_{12} \\
2_{12}-1_{01} \\
3_{03}-2_{12} \\
4_{14}-3_{03} \\
2_{21}-1_{10} \\
2_{11}-2_{02} \\
2_{02}-1_{11} \\
1_{11}-0_{00} \\
2_{20}-2_{11} \\
3_{22}-3_{13} \\
3_{13}-2_{02}\end{array}$ & $\begin{array}{r}1097.365 \\
1162.912 \\
1661.008 \\
1669.905 \\
1716.769 \\
2640.474 \\
2773.978 \\
752.033 \\
987.927 \\
1113.343 \\
1228.789 \\
1919.359 \\
2164.132\end{array}$ & $\begin{array}{c}215.1 \\
271.0 \\
159.8 \\
80.1 \\
162.5 \\
289.2 \\
159.8 \\
136.9 \\
100.8 \\
53.4 \\
195.9 \\
296.8 \\
204.7\end{array}$ & $\begin{array}{c}18.7 \\
17.1 \\
12.7 \\
12.7 \\
12.3 \\
8.0 \\
7.6 \\
33.4 \\
19.8 \\
18.7 \\
17.6 \\
9.8 \\
11.5\end{array}$ & $\begin{array}{c}4.15 \\
5.80 \\
4.8 \\
14.3 \\
6.9 \\
5.1^{\mathrm{b}} \\
6.5 \\
3.42 \\
6.09 \\
3.49 \\
5.30 \\
2.4 \\
6.9\end{array}$ & $\begin{array}{l}0.03 \\
0.14 \\
\ldots \\
\ldots \\
\ldots \\
\ldots \\
\ldots \\
0.07 \\
0.11 \\
0.05 \\
0.09 \\
\ldots \\
\cdots\end{array}$ & $\begin{array}{c}0.42 \\
0.60 \\
1.6 \\
4.3 \\
2.2 \\
1.6 \\
2.1 \\
0.35 \\
0.62 \\
0.35 \\
0.54 \\
0.8 \\
2.2\end{array}$ & $\begin{array}{l}2 \\
2 \\
3 \\
3 \\
3 \\
3 \\
3 \\
2 \\
2 \\
2 \\
2 \\
3 \\
3\end{array}$ \\
\hline $\begin{array}{l}\mathrm{O}-\mathrm{H}_{2} \mathrm{O}^{+} \\
\mathrm{o}-\mathrm{H}_{2} \mathrm{O}^{+} \\
\mathrm{OH}^{+} \\
\mathrm{CH}^{+} \\
\mathrm{HF}\end{array}$ & $\begin{array}{ll}1_{11}-0_{00} & \mathrm{~J}=3 / 2-1 / 2 \\
1_{11}-0_{00} & \mathrm{~J}=1 / 2-1 / 2 \\
1_{12}-0_{12} & \\
\mathrm{~J}=1-0 & \\
\mathrm{~J}=1-0 & \end{array}$ & $\begin{array}{c}1115.204 \\
1139.561 \\
1033.118 \\
835.079 \\
1232.476\end{array}$ & $\begin{array}{l}53.5 \\
53.5 \\
49.6 \\
39.6 \\
59.1\end{array}$ & $\begin{array}{l}18.7 \\
17.1 \\
18.7 \\
35.0 \\
17.6\end{array}$ & $\begin{array}{l}2.13 \\
1.98 \\
4.34 \\
3.16 \\
2.16\end{array}$ & $\begin{array}{l}0.05 \\
0.09 \\
0.16 \\
0.08 \\
0.09\end{array}$ & $\begin{array}{l}0.22 \\
0.22 \\
0.46 \\
0.33 \\
0.23\end{array}$ & $\begin{array}{l}2 \\
2 \\
2 \\
2 \\
2\end{array}$ \\
\hline$[\mathrm{NII}]$ & ${ }^{3} P_{1}-{ }^{3} P_{0}$ & 1461.134 & 6.77 & 16.8 & 54.97 & 1.42 & 5.67 & 2 \\
\hline & itended source & & & & & & & \\
\hline${ }^{12} \mathrm{CO}$ & $\begin{array}{l}\mathrm{J}=2-1 \\
\mathrm{~J}=3-2 \\
\mathrm{~J}=4-3 \\
\mathrm{~J}=5-4 \\
\mathrm{~J}=6-5 \\
\mathrm{~J}=7-6 \\
\mathrm{~J}=8-7\end{array}$ & $\begin{array}{l}230.538 \\
345.796 \\
461.041 \\
576.268 \\
691.473 \\
806.652 \\
921.800\end{array}$ & $\begin{array}{c}16.60 \\
33.19 \\
55.32 \\
82.97 \\
116.16 \\
154.87 \\
199.11\end{array}$ & \begin{tabular}{l|l}
31.0 & \\
43.4 & \\
43.4 & \\
43.4 \\
43.4 \\
43.4 \\
43.4 & \\
\end{tabular} & $\begin{array}{c}6.41 \\
19.4 \\
21.69 \\
26.80 \\
24.73 \\
21.06 \\
19.78\end{array}$ & $\begin{array}{l}.015 \\
\cdots \\
0.66 \\
0.43 \\
0.24 \\
1.30 \\
0.64\end{array}$ & \begin{tabular}{c|} 
\\
1.3 \\
3.4 \\
2.73 \\
3.30 \\
3.02 \\
2.88 \\
2.50
\end{tabular} & $\begin{array}{l}4 \\
5 \\
6 \\
6 \\
6 \\
6 \\
6\end{array}$ \\
\hline$[\mathrm{CI}]$ & $\begin{array}{l}{ }^{3} P_{1}-{ }^{3} P_{0} \\
{ }^{3} P_{2}-{ }^{3} P_{1}\end{array}$ & $\begin{array}{l}492.161 \\
809.342\end{array}$ & $\begin{array}{l}23.62 \\
62.46\end{array}$ & $\begin{array}{l}43.4 \\
43.4\end{array}$ & $\begin{array}{l}12.81 \\
21.08\end{array}$ & $\begin{array}{l}0.13 \\
1.20\end{array}$ & $\begin{array}{l}1.57 \\
2.84\end{array}$ & $\begin{array}{l}6 \\
6\end{array}$ \\
\hline$[\mathrm{NII}]$ & ${ }^{3} P_{1}-{ }^{3} P_{0}$ & 1461.134 & 6.77 & 43.4 & 187.9 & 8.2 & 24.3 & 6 \\
\hline
\end{tabular}

REFERENCES. - (1): Interferometric observations from Krips et al. (2011), adding together the emission from the E-knot and the W-knot and using the conversion: $f\left(\mathrm{~W} \mathrm{~m}^{-2}\right)=3.33 \times 10^{-23} \nu_{G H z} S_{C O}\left(\mathrm{Jy} \mathrm{km} \mathrm{s}^{-1}\right) ;(2)$ : This work, SPIRE-FTS deep pointed spectrum; (3): S. Hailey-Dunsheath, 2012, private communication; (4): From CSO Z-Spec observations of Kamenetzky et al. 2011, using the same conversion as for (1); (5): C. Wilson, 2012, private communication; (6): This work, SPIRE-FTS line maps

NotE. - ${ }^{a}$ This line is possibly contaminated by the o- $\mathrm{H}_{2} \mathrm{O} 33_{12}-2_{21}$ at $1153.127 \mathrm{GHz}$. However, the line fitting results indicate that the $\mathrm{CO}$ line is dominant; therefore we assign the whole flux to the $\mathrm{CO}$ line; ${ }^{\mathrm{b}}$ This line is blended with the $\mathrm{CO}(23-22)$ transition, so to obtain the flux of water we subtracted the flux of $\mathrm{CO}(23-22)$ predicted by the model presented by Hailey-Dunsheath et al. (2012). 
TABLE 2

RADEX Model Parameters and Ranges

\begin{tabular}{ccc}
\hline \hline Parameter & Range & \# of Points \\
\hline$T_{\text {kin }}[\mathrm{K}]$ & $10^{1.7}-10^{3.5}$ & 90 \\
$n\left(\mathrm{H}_{2}\right)\left[\mathrm{cm}^{-3}\right]$ & $10^{1.0}-10^{5.0}$ & 90 \\
$N_{\mathrm{CO}}\left[\mathrm{cm}^{-2}\right]$ & $10^{16}-10^{20}$ & 90 \\
$\Delta V\left[\mathrm{~km} \mathrm{~s}^{-1}\right]$ & 1.0 & fixed \\
\hline
\end{tabular}

Note. - All parameters are sampled evenly in $\log$ space.

TABLE 3

Multi-Component Radiative Transfer Results

\begin{tabular}{|c|c|c|c|c|}
\hline Parameter & Median & $1 \sigma$ Range & 1D Max & 4D Max \\
\hline \multicolumn{5}{|l|}{ compact source } \\
\hline $\begin{array}{c}T_{\mathrm{kin}}[\mathrm{K}] \\
n\left(\mathrm{H}_{2}\right)\left[\mathrm{cm}^{-3}\right] \\
N_{C O}\left[\mathrm{~cm}^{-2}\right] \\
\Phi_{\mathrm{A}} \\
P\left[\mathrm{~K} \mathrm{~cm}^{-2}\right] \\
\left\langle N_{\mathrm{CO}}\right\rangle\left[\mathrm{cm}^{-2}\right] \\
\mathrm{M}\left(\mathrm{H}_{2}\right)\left[\mathrm{M}_{\odot}\right]\end{array}$ & $\begin{array}{c}92 \\
10^{4.6} \\
10^{19.5} \\
10^{-0.9} \\
10^{6.6} \\
10^{18.6} \\
10^{7.4}\end{array}$ & $\begin{array}{c}74-120 \\
10^{4.4}-10^{4.8} \\
10^{19.3}-10^{19.7} \\
10^{-1.0}-10^{-0.9} \\
10^{6.5}-10^{6.8} \\
10^{18.4}-10^{18.7} \\
10^{7.2}-10^{7.5}\end{array}$ & $\begin{array}{c}87 \\
10^{4.6} \\
10^{19.5} \\
10^{-0.9} \\
10^{6.6} \\
10^{18.6} \\
10^{7.4}\end{array}$ & $\begin{array}{c}100 \\
10^{4.5} \\
10^{19.6} \\
10^{-0.9} \\
10^{6.6} \\
10^{18.6} \\
10^{7.4}\end{array}$ \\
\hline \multicolumn{5}{|l|}{ extended source } \\
\hline $\begin{array}{c}T_{\text {kin }}[\mathrm{K}] \\
n\left(\mathrm{H}_{2}\right)\left[\mathrm{cm}^{-3}\right] \\
N_{C O}\left[\mathrm{~cm}^{-2}\right] \\
\Phi_{\mathrm{A}} \\
P\left[\mathrm{~K} \mathrm{~cm}^{-2}\right] \\
\left\langle N_{\mathrm{CO}}\right\rangle\left[\mathrm{cm}^{-2}\right] \\
\mathrm{M}\left(\mathrm{H}_{2}\right)\left[\mathrm{M}_{\odot}\right]\end{array}$ & $\begin{array}{c}116 \\
10^{2.8} \\
10^{18.8} \\
10^{-1.2} \\
10^{4.7} \\
10^{17.7} \\
10^{8.5}\end{array}$ & $\begin{array}{c}80-166 \\
10^{2.3}-10^{3.3} \\
10^{18.3}-10^{19.3} \\
10^{-1.3}-10^{-1.0} \\
10^{4.3}-10^{5.2} \\
10^{17.2}-10^{18.2} \\
10^{8.0}-10^{9.0}\end{array}$ & $\begin{array}{c}121 \\
10^{2.9} \\
10^{18.7} \\
10^{-1.2} \\
10^{4.4} \\
10^{17.6} \\
10^{8.5}\end{array}$ & $\begin{array}{c}127 \\
10^{2.9} \\
10^{18.7} \\
10^{-1.1} \\
10^{4.4} \\
10^{17.6} \\
10^{8.5}\end{array}$ \\
\hline
\end{tabular}

TABLE 4

THE BEST FIT MODEL PARAMETERS OF $\mathrm{H}_{2} \mathrm{O}$

\begin{tabular}{cc}
\hline \hline Parameter & Value \\
\hline $\begin{array}{c}\text { Temperature } \\
\text { density }\end{array}$ & $40 \pm 10 \mathrm{~K}$ \\
diameter & $10^{6}-8 \times 10^{7} \mathrm{~cm}^{-3}$ \\
$N\left(\mathrm{o}-\mathrm{H}_{2} \mathrm{O}\right)$ & $2 \times 10^{17} \pm 2^{\prime \prime}$ \\
$\mathrm{N}\left(\mathrm{p}-\mathrm{H}_{2} \mathrm{O}\right)$ & $5 \times 10^{16} \mathrm{~cm}^{-2}$ \\
& \\
\hline
\end{tabular}

